\title{
Classical and Quantum-Mechanical Systems of Toda Lattice Type. I
}

\author{
Roe Goodman ${ }^{1}$ and Nolan R. Wallach ${ }^{2}$ \\ Department of Mathematics, Rutgers University, New Brunswick, NJ 08903, USA
}

\begin{abstract}
The structure of the commutant of Laplace operators in the enveloping and "Poisson algebra" of certain generalized " $a x+b$ " groups leads (in this article) to a determination of classical and quantum mechanical first integrals to generalized periodic and non-periodic Toda lattices. Certain new Hamiltonian systems of Toda lattice type are also shown to fit in this framework. Finite dimensional Lax forms for the (periodic) Toda lattices are given generalizing results of Flaschke.
\end{abstract}

\section{Contents}

0. Introduction . . . . . . . . . . . . . . . . . . . . . . . . . . . . . 355

1. Factorization of the Harish-Chandra Homomorphism . . . . . . . . . . . . . . . . 359

2. Centralizers of Laplacians . . . . . . . . . . . . . . . . . . . . . . . . . . . . 361

3. Inversion of the Symbol Map . . . . . . . . . . . . . . . . . . . . . . . . . . . 366

4. Structure of $U(\mathfrak{b})^{\Omega}$ for Dynkin Diagrams . . . . . . . . . . . . . . . . . . . . . . . . 369

5. Structure of $U(\mathfrak{b})^{\Omega}$ for Extended Dynkin Diagrams . . . . . . . . . . . . . . . . . . . . . . . 371

6. Centralizer of the Laplacian in the Poisson Algebra . . . . . . . . . . . . . . . . . 373

7. Cotangent Bundles and Coadjoint Orbits . . . . . . . . . . . . . . . . . . . . . . 376

8. Lax Forms . . . . . . . . . . . . . . . . . . . . . . . . . . . . . . . . . . . 380

9. Complete Integrability via Lax Forms . . . . . . . . . . . . . . . . . . . . . . . 384

References . . . . . . . . . . . . . . . . . . . . . . . . . . . 386

\section{Introduction}

In this paper we study the Laplace operator and its commutant on a class of generalized " $a x+b$ " Lie groups. This analysis is carried out both in the universal enveloping algebra of the Lie algebra and in the "Poisson algebra". The problem in the Poisson algebra amounts to finding first integrals for certain Hamiltonian systems (which generalize both the periodic and non-periodic Toda lattices). The

I Research partially supported by NSF grant MCS 79-03223

2 Research partially supported by NSF grant MCS 79-03153 
problem in the enveloping algebra amounts to finding quantum mechanical first integrals for the quantized versions of these Hamiltonian systems. For the generalized non-periodic Toda lattices (as studied most profoundly by Kostant [12]) and all but four of the generalized periodic Toda lattices (Adler and van Moerbeke [2]), we show that the two problems are essentially identical. Also in the Poisson algebra case we show that there is an infinite sequence of new integrable Hamiltonian systems that do not correspond to completed Dynkin diagrams in the sense of Bourbaki [6]. (They do however correspond to Coxeter diagrams of finite groups generated by reflections.)

The four generalized periodic Toda lattices that do not fit into our uniform quantum and classical mechanical techniques are handled in this article using Lax forms. We derive in particular a finite-dimensional Lax form (generalizing that of Flaschka [7] for the periodic Toda lattice) for all first integrals of all the periodic Toda lattices associated with completed Dynkin diagrams. In the case of nonperiodic Toda lattices this Lax form immediately shows that the full system of first integrals can be linearized in terms of the action of $A$ on $G / M A N$ for $G$ the corresponding real, split, semi-simple Lie group and $M A N$ a minimal parabolic subgroup. In the case of the periodic Toda lattice this is also true, with $G$ replaced by a certain infinite-dimensional Lie group. These results will be the topic of the second paper in this series. Also in a forthcoming paper will be another infinite family of integrable Hamiltonian systems associated with certain "non-generic" orbits of $A$ on $G / M A N$.

The joint spectral decomposition of the quantum first integrals of the quantized Toda lattices will be the subject of a later paper in this series. In the case of the non-periodic Toda lattices this is intimately connected with the work of Kostant [11] and Goodman and Wallach [8] on Whittaker vectors. For the general case the existence of the joint spectral decomposition is guaranteed by the results of this paper (commutativity of the centralizer of the Laplacian in the enveloping algebra) and a theorem of Nelson and Stinespring [15].

To describe our results and motivation in more detail, consider the class of solvable Lie algebras over $\mathbb{R}$ of the form

$$
\mathfrak{b}=\mathfrak{a} \oplus \mathfrak{u},
$$

where $\mathfrak{a}$ and $\mathfrak{u}$ are commutative subalgebras, with $\mathfrak{u}$ an ideal. Assume that there is an inner product $\langle\cdot, \cdot\rangle$ on $\mathfrak{b}$ so that $(0.1)$ is an orthogonal decomposition and $\operatorname{ad}(H), H \in \mathfrak{a}$, is self-adjoint. Let $\Omega=\sum H_{i}^{2}+\sum X_{j}^{2}$, where $\left\{H_{i}\right\}$ and $\left\{X_{j}\right\}$ are orthonormal bases for $\mathfrak{a}$ and $\mathfrak{u}$, respectively. Thus $\Omega$ is an elliptic operator in the enveloping algebra $U(\mathfrak{b})$, which we shall call the Laplacian of $\mathfrak{b}$. The principal objects studied in this paper are the centralizers of $\Omega$ in $U(\mathfrak{b})$ and $S(\mathfrak{b})$ (the symmetric tensor algebra with its canonical Poisson algebra structure).

Let $g$ (respectively $G$ ) be a semi-simple Lie algebra (respectively Lie group) over $R$, and $\mathfrak{g}=\mathfrak{f} \oplus \mathfrak{a} \oplus \mathfrak{n}$ (respectively $G=K A N$ ) an Iwasawa decomposition. Our original motivation comes from the case when $\mathfrak{b}=\mathfrak{a} \oplus \mathrm{n} /[\mathrm{n}, \mathrm{n}]$, with $\langle\cdot, \cdot\rangle$ induced by projecting the Killing form, $B$, from $\mathfrak{f}^{\perp}$ onto $\mathfrak{a} \oplus \mathrm{n}$. In this case, letting $U(\mathfrak{g})^{\mathfrak{f}}$ denote the centralizer of $\mathfrak{f}$ in $U(\mathfrak{g})$, we construct in Sect. 1 a canonical homomorphism

$$
\tilde{\gamma}: U(\mathfrak{g})^{\mathfrak{f}} \rightarrow U(\mathfrak{b}),
$$


so that if $C$ is the Casimir operator of $g$ defined by $B$, then $\tilde{\gamma}(C)=\Omega+$ const. Since $C$ is central in $U(\mathrm{~g})$, it is clear that

$$
\tilde{\gamma}\left(U(\mathfrak{g})^{\mathfrak{f}}\right) \leqq U(\mathfrak{b})^{\Omega},
$$

where $U(\mathfrak{b})^{\Omega}$ is the centralizer of $\Omega$. In Sect. 4 we prove that when $G$ is split, one has equality in $(0.2)$, and $U(\mathfrak{b})^{\Omega}$ is isomorphic to a polynomial algebra in $\operatorname{dim}(\mathfrak{a})$ generators.

The differential equations satisfied by Whittaker functions on $A$ associated with the spherical principal series $\pi_{v}$ (Kostant [11] and Goodman and Wallach [8]), are given as

$$
\pi(\tilde{\gamma}(u)) f=\chi_{v}(u) f,
$$

where $\pi$ is a fixed representation of $\mathfrak{b}$ on $C^{\infty}(\mathfrak{a})$ (see below), and $\chi_{v}$ is the character of $U(\mathfrak{g})^{\mathfrak{f}}$ associated with $v$. In light of $(0.3)$, the results of Sect. 4 amount to an algorithm for describing explicitly the differential equations satisfied by Whittaker functions on $G$. The application of this analysis will appear in a later paper.

Return to the case of a general algebra $\mathfrak{b}$ as above, with corresponding simplyconnected Lie group $B$. Using the standard Kirillov-Kostant-Souriau symplectic structure on $\mathfrak{b}^{*}$, one finds that $\Omega$ corresponds on generic orbits of $B$ on $\mathfrak{b}^{*}$ to a Hamiltonian system on $\mathfrak{a} \times \mathfrak{a}^{*}$ with Hamiltonian

$$
H=\sum p_{i}^{2}+\sum_{j=1}^{m} c_{j}^{2} e^{-2 \alpha_{j}} .
$$

(Here $p_{i}$ are orthogonal coordinates on $\mathfrak{a}^{*}, c_{j} \neq 0$ depend on the orbit, and $\left\{\alpha_{j}\right\}$ is the set of roots of $\mathfrak{a}$ on 11.) These systems contain as special cases all of the "generalized Toda lattices" (non-periodic and periodic). The elements of $U(\mathfrak{b})^{\Omega}$ then define first integrals of $H$ via symmetrization from $U(\mathfrak{b})$ to $S(\mathfrak{b})$.

We define a representation $\pi$ of $\mathfrak{b}$ on $C^{\infty}(\mathfrak{a})$ by

$$
\begin{gathered}
\pi\left(H_{i}\right) f(x)=\left.\frac{d}{d t} f\left(x-t H_{i}\right)\right|_{t=0}, \\
\pi\left(X_{j}\right) f(x)=i c_{j} e^{-\alpha_{j}(x)} f(x)
\end{gathered}
$$

for $f \in C^{\infty}(\mathfrak{a})$, where the $X_{j}$ satisfy the commutation relations $\left[Y, X_{j}\right]=\alpha_{j}(Y) X_{j}$ for $Y \in \mathfrak{a}$. Then

$$
\pi(\Omega)=\Delta-\sum_{j=1}^{m} c_{j}^{2} e^{-2 \alpha_{j}}
$$

with $\Delta$ the Laplacian on $\mathfrak{a}$ associated with $\left.\langle\cdot, \cdot\rangle\right|_{\mathfrak{a} \times \mathfrak{a}}$. Formula $(0.5)$ is the usual quantization of (0.4), and in this case the elements $\pi(T), T \in U(\mathfrak{b})^{\Omega}$, now become quantum first integrals of $H$.

Let $\mu: \mathfrak{b} \rightarrow \mathfrak{a}$ be the projection corresponding to the decomposition $\mathfrak{b}=\mathfrak{a} \oplus \mathfrak{u}$, and extend $\mu$ to a homomorphism of $U(\mathfrak{b})$ to $U(\mathfrak{a})$. Let $W$ be the group of orthogonal transformations of $\mathfrak{a}$ generated by the reflections about the hyperplanes $\alpha_{j}=0$. [One knows that the ring of invariants $U(\mathfrak{a})^{W}$ is a polynomial algebra in $\ell=\operatorname{dim}(\mathfrak{a})$ generators precisely when $W$ is a finite group.] Our main results in this paper are the following: 
(I) $\mu\left(U(\mathfrak{b})^{\Omega}\right) \cong U(\mathfrak{a})^{W}$ (Theorem 2.6).

(II) If $\alpha_{1}, \ldots, \alpha_{n}$ are linearly independent, then $\mu: U(\mathfrak{b})^{\Omega} \rightarrow U(\mathfrak{a})^{W}$ is injective. In particular, $U(\mathfrak{b})^{\Omega}$ is commutative in this case (Theorem 2.5).

Note that combining (I) and (II) with the Chevalley restriction theorem gives equality in $(0.2)$ for the non-periodic generalized Toda lattices treated, e.g. by Kostant [12], and hence establishes their complete integrability, in the generic sense, Sect. 4. (Kostant's results, however, are more precise concerning the independence of the basic invariants at every point of a generic coadjoint orbit in $\mathfrak{b}^{*}$.)

(III) Suppose the set of roots $\alpha_{1}, \ldots, \alpha_{m}$ forms a completed Dynkin diagram (in the sense of Bourbaki [6]), or corresponds to the diagram $C_{\ell}$ in Fig. $5.1(m=\ell+1)$. With the possible exception of the systems associated with $E_{7}, E_{8}, G_{2}$, or $F_{4}$, one has $\mu(U(\mathfrak{b}))^{\Omega}=U(\mathfrak{a})^{W}$ (Theorem 5.2).

(IV) With $\alpha_{1}, \ldots, \alpha_{m}$ as in (III), $U(\mathfrak{b})^{\Omega}$ is a polynomial algebra in $\ell+1$ commuting generators (Theorem 5.2).

The analogues of (I)-(IV) are also true in the Poisson algebra $S(\mathrm{~b})$ (Sect. 6; the diagram $B_{\ell}$ from Fig. 5.1 is also allowed now). The proofs of (III) and (IV) give an explicit inductive procedure for constructing generators of $U(\mathfrak{b})^{\Omega}$, starting with generators for $U(\mathfrak{a})^{W}$.

The results just cited establish the equivalence between the classical and quantum mechanical integrability of the systems $(0.4)$ and $(0.5)$.

The exceptional completed Dynkin diagrams excluded in (III) and (IV), for the case of the Poisson algebra, are treated in Sects. 8 and 9 via Lax forms. It should be noted that the existence of "Lax forms" for first integrals of $H$ is not sufficient to establish Poisson commutativity of these functions. For this we also need the results of Sects. 3 and 6 .

For references to the extensive literature on "generalized Toda Lattices", see Adler [1], Adler and van Moerbeke [2], Kostant [12], Moser [13], and Olshanetsky and Perelomov [16]. General Hamiltonians of the form (0.4) seem to have been first considered by Bogoyavlensky [5], who points out the connection between the finiteness of the group $W$ and the existence of a complete set of invariants of motion of the system. Our formulas for the basic invariants, in the case of the original "Toda Lattice", are related to the calculations of Hénon [10]. In our approach, the surjectivity of the Harish-Chandra homomorphism and the Chevalley restriction theorem for the invariant differential operators on a symmetric space [9] serve as the underlying existence theorems for complete integrability (in both the quantum and classical mechanical sense). The role of the additional invariant [a generator for the center of $U(b)]$, which occurs in the study of the periodic systems, was also noted by Bogoyavlensky [5] and Adler and van Moerbeke [2].

The calculation of the symplectic structure and coadjoint orbits in Sect. 7 is standard. The formula (7.9) for the Hamiltonian vector field corresponding to a left-invariant function on the cotangent bundle of a Lie group appears first in Arnol'd [3] (Miščenko-Fomenko [14]). This gives the link between the enveloping algebra-Poisson algebra calculations of Sects. 2-6 and the Lax forms in Sect. 8. The complete integrability of the generalized periodic Toda Lattice has also been obtained by Adler and van Moerbeke, [2], using Kac-Moody Lie algebras (Ratiu 
[17]). Our proof, on the other hand, stays entirely within the category of finitedimensional Lie groups, and also applies to the quantized systems (ignoring four exceptional diagrams).

\section{Factorization of the Harish-Chandra Homomorphism}

Let $G$ be a real, connected semi-simple Lie group with finite center, and fix an Iwasawa decomposition $G=N A K$ ( $N$ nilpotent, $A \simeq \mathbb{R}^{\ell}, K$ maximal compact subgroup). Let $\mathfrak{g}, \mathfrak{n}, \mathfrak{a}, \mathfrak{f}$ denote the Lie algebras of the corresponding groups. Then $\mathfrak{g}=\mathfrak{n}+\mathfrak{a}+\mathfrak{f}$ (vector space direct sum). Set

$$
\mathfrak{s}=\mathfrak{a}+\mathfrak{n} .
$$

Then $\mathfrak{s}$ is a solvable subalgebra of $\mathfrak{g}$, and by the Poincaré-Birkhoff-Witt (PBW.) theorem, one has a direct sum decomposition

$$
U(\mathfrak{g})=U(\mathfrak{s}) \oplus\{U(\mathfrak{g}) \mathfrak{f}\} .
$$

Let

$$
p: U(\mathfrak{g}) \rightarrow U(\mathfrak{s})
$$

be the projection defined by (1.1).

Lemma 1.1. If $x \in U(\mathfrak{g})$ and $y \in U(\mathfrak{g})^{\mathfrak{f}}$, then $p(x y)=p(x) p(y)$. In particular, the restriction of $p$ to $U(\mathrm{~g})^{\mathfrak{f}}$ is a homomorphism.

Proof. Clearly $x y \equiv p(x) y \quad \bmod U(\mathfrak{g}) \mathfrak{f}$, since $y$ commutes with $\mathfrak{f}$, while $p(x) y \equiv p(x) p(y) \bmod U(\mathfrak{g}) \mathfrak{f}$, since $U(\mathfrak{g}) \mathfrak{f}$ is a left ideal in $U(\mathfrak{g})$. Combining these two observations gives the lemma.

The derived algebra $[n, n]$ of $n$ is an ideal in $\mathfrak{s}$. We set $\mathfrak{b}=\mathfrak{s} /[n, n]$ and let

$$
\pi: U(\mathfrak{s}) \rightarrow U(\mathfrak{b})
$$

be the canonical quotient homomorphism. Writing $\mathfrak{u}=\mathfrak{n} /[\mathfrak{n}, \mathfrak{n}]$, we have $\mathfrak{b}=\mathfrak{a}+\mathfrak{u}$. If $A^{+} \subset \mathfrak{a}^{*}$ is the set of roots for the action of $\operatorname{ad}(\mathfrak{a})$ on $\mathfrak{n}$, and $\left\{\alpha_{1}, \ldots, \alpha_{\ell}\right\} \subset A^{+}$is the set of simple roots, then

$$
\mathfrak{u}=\sum_{i=1}^{\ell} \mathfrak{u}_{\alpha_{i}},
$$

where $\mathfrak{u}_{\alpha}=\{X \in \mathfrak{u}:[H, X]=\alpha(H) X$ for $H \in \mathfrak{a}\}$. Thus the only nontrivial commutation relations in $\mathfrak{b}$ are

$$
[H, X]=\alpha_{i}(H) X,
$$

for $H \in \mathfrak{a}, X \in \mathfrak{u}_{\alpha_{i}}, 1 \leqq i \leqq \ell$.

The isomorphism $\mathfrak{a} \cong \mathfrak{b} / \mathfrak{u}$ induces a homomorphism

$$
\mu: U(\mathfrak{b}) \rightarrow U(\mathfrak{a})
$$


Set $q=\mu_{\circ} \pi_{\circ} p$, so that the diagram

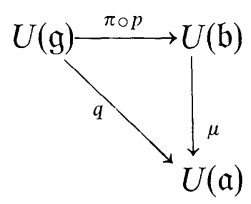

is commutative. From Lemma 1.1 one has

$$
q(x y)=q(x) q(y), x \in U(\mathfrak{g}), y \in U(\mathfrak{g})^{\mathfrak{f}} .
$$

Define as usual

$$
\varrho(H)=(1 / 2) \operatorname{tr}\left(\left.\operatorname{ad}(H)\right|_{\mathfrak{n}}\right), \quad \text { for } H \in \mathfrak{a},
$$

and let $\tau: U(\mathfrak{a}) \rightarrow U(\mathfrak{a})$ be the automorphism such that

$$
\tau(H)=H+\varrho(H) 1, \quad H \in \mathfrak{a} .
$$

We may extend $\tau$ to an automorphism $\tilde{\tau}$ of $U(\mathfrak{b})$ by setting

$$
\tilde{\tau}(H+X)=\tau(H)+X, \text { for } H \in \mathfrak{a}, X \in \mathfrak{u} .
$$

Define $\gamma=\tau \circ q$ and $\tilde{\gamma}=\tilde{\tau} \circ \pi \circ p$. Since $\mu \circ \tilde{\tau}=\tau$, we have the commutative diagram

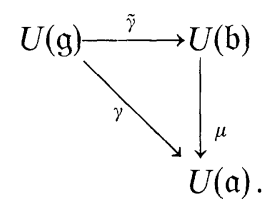

The restriction of $\gamma$ to $U(\mathfrak{g})^{\mathfrak{f}}$ is the celebrated "Harish-Chandra homomorphism" [9, Chap. X, Sect. 6.3]. Let $W$ be the Weyl group of $(\mathfrak{g}, \mathfrak{a})$. One knows that

$$
\gamma: U(\mathfrak{g})^{\mathfrak{f}} \rightarrow U(\mathfrak{a})^{W}
$$

is surjective.

Lemma 1.2. $\mu$ maps $\tilde{\gamma}\left(U(\mathfrak{g})^{\mathfrak{f}}\right)$ onto $U(\mathfrak{a})^{W}$.

Proof. Immediate by (1.3).

Let $\theta$ be the Cartan involution on $\mathfrak{g}$ associated with $\mathfrak{f}$, and

$$
\mathfrak{g}=\mathfrak{l}+\mathfrak{p}
$$

the Cartan decomposition. Define $\sigma(X)=(X-\theta X) / 2$, so that

$$
\sigma: \mathfrak{g} \rightarrow \mathfrak{p}
$$

is the projection onto $\mathfrak{p}$. The Cartan-Killing form $B$ is positive-definite on $\mathfrak{p}$. Since the restriction of $\sigma$ to $\mathfrak{s}$ is bijective, we obtain a positive-definite inner product on $\mathfrak{s}$ by setting

$$
\langle X, Y\rangle=B(\sigma(X), \sigma(Y))
$$

for $X, Y \in \mathfrak{s}$. 
Lemma 1.3. The decomposition

$$
\mathfrak{s}=\mathfrak{a} \oplus \sum_{\alpha>0} \mathrm{n}_{\alpha}
$$

is orthogonal relative to $\langle\cdot, \cdot\rangle$. If one writes $X=X_{\mathfrak{a}}+X_{+}$, where $X_{\mathfrak{a}} \in \mathfrak{a}$ and $X_{+} \in \mathfrak{n}$, then for $X, Y \in \mathfrak{s}$,

$$
\langle X, Y\rangle=B\left(X_{\mathfrak{a}}, Y_{\mathfrak{a}}\right)-(1 / 2) B\left(X_{+}, \theta\left(Y_{+}\right)\right) .
$$

Proof. Straightforward calculation.

We continue to denote by $\langle\cdot, \cdot\rangle$ the quotient inner product on $\mathfrak{b}$ induced by the inner product on $\mathfrak{s}$. Note that by Lemma 1.3 , the orthogonal complement of $[n, n]$ in $\mathfrak{s}$ is

$$
\mathfrak{a} \oplus \sum_{i=1}^{\ell} \mathrm{n}_{\alpha_{i}},
$$

which is thus isomorphic to $\mathfrak{b}$ as a Euclidean vector space.

Proposition 1.4. Let $C$ be the Casimir operator in $U(\mathfrak{g})$, defined via the CartanKilling form. Suppose $\left\{h_{i}\right\}$ is an orthonormal basis for $\mathfrak{a}$ and $\left\{X_{i j}\right\}_{1 \leqq j \leqq m_{i}}$ is an orthonormal basis for $\mathfrak{u}_{\alpha_{i}}$. Then

$$
\tilde{\gamma}(C)=\sum_{i=1}^{\ell} h_{i}^{2}+\sum_{i=1}^{\ell} \sum_{j=1}^{m_{i}} X_{i j}^{2}-\langle\varrho, \varrho\rangle .
$$

Proof. Let $\mathfrak{v}=\theta \mathfrak{n}$ be the opposed algebra to $\mathfrak{n}$, and let $\mathfrak{m}$ be the centralizer of $\mathfrak{a}$ in $\mathfrak{f}$. Then $\mathfrak{g}=\mathfrak{n} \oplus \mathfrak{a} \oplus \mathfrak{m} \oplus \mathfrak{v}$. Enumerate $\Lambda^{+}$as $\left\{\alpha_{1}, \ldots, \alpha_{d}\right\}$. For $1 \leqq i \leqq d$ pick a basis $\left\{Z_{i j}\right\}_{1 \leqq j \leqq m_{i}}$ for $\mathrm{n}_{\alpha_{i}}$, such that $\pi\left(Z_{i j}\right)=X_{i j}$ when $1 \leqq i \leqq \ell$ and $B\left(Z_{i j}, \theta Z_{m n}\right)=-2 \delta_{i m} \delta_{j n}$. [This is the correct normalization of $Z_{i j}$, by formula (1.4).] Then setting $Y_{i j}=-(1 / 2) \theta Z_{i j}$, one has

$$
C=\sum_{i=1}^{\ell} h_{i}^{2}+\sum_{i=1}^{d} \sum_{j=1}^{m_{2}}\left(Z_{i j} Y_{i j}+Y_{i j} Z_{i j}\right)+C_{\mathfrak{m}} \text {, }
$$

where $C_{\mathrm{m}} \in U(\mathrm{~m})$.

Now $\left[X_{i j}, Y_{i j}\right]=H_{\alpha_{i}}$, where $B\left(H_{\alpha}, H\right)=\alpha(H)$ for all $H \in \mathfrak{a}$. Since $Y_{i j} \equiv(1 / 2) X_{i j}$ modf́, and $2 \varrho=\sum_{i=1}^{d} m_{i} \alpha_{i}$, this gives

$$
C \equiv \sum_{i=1}^{\ell} h_{i}^{2}+\sum_{i=1}^{d} \sum_{j=1}^{m_{i}} X_{i j}^{2}-2 H_{\varrho}
$$

$\bmod U(\mathfrak{g}) \mathfrak{f}$. Projecting onto $U(\mathfrak{b})$ and applying the automorphism $\tilde{\tau}$, we obtain (1.5).

\section{Centralizers of Laplacians}

With the results of Sect. 1 as a model, we consider a finite-dimensional real Lie algebra $\mathfrak{b}$, equipped with a positive-definite inner product $\langle\cdot, \cdot\rangle$, such that

(i) $\mathfrak{b}=\mathfrak{a} \oplus \mathfrak{u}$ (orthogonal direct sum), with $[\mathfrak{a}, \mathfrak{u}] \subseteq \mathfrak{u}$ and both $\mathfrak{a}$ and $\mathfrak{u}$ abelian;

(ii) for $H \in \mathfrak{a}, \operatorname{ad}(H)$ is symmetric, relative to $\langle\cdot, \cdot\rangle$. 
By (i) and (ii) we may simultaneously diagonalize the action of $\mathfrak{a}$ on $\mathfrak{u}$. Let $X_{1}, \ldots, X_{m}$ be an orthonormal basis for $\mathfrak{u}$ such that

$$
\left[H, X_{i}\right]=\alpha_{i}(H) X_{i}, \quad H \in \mathfrak{a} .
$$

Here $\alpha_{i} \in \mathfrak{a}^{*}$, and (2.1) is the only non-trivial commutation relation in $\mathfrak{b}$. We shall refer to $\left\{\alpha_{1}, \ldots, \alpha_{m}\right\}$ as the roots of $\mathfrak{a}$ on $\mathfrak{u}$.

Let $\left\{W_{i}\right\}$ be any basis for $\mathfrak{b}$. If $c_{i j}=\left\langle W_{i}, W_{j}\right\rangle$ and $\left[c^{i j}\right]$ is the matrix inverse to $\left[c_{i j}\right]$, then the element

$$
\Omega=\sum c^{i j} W_{i} W_{j}
$$

of $U(\mathfrak{b})$ is independent of the choice of $\left\{W_{i}\right\}$, and will be called the Laplacian of $\mathfrak{b}$ (relative to the given inner product $\langle\cdot, \cdot\rangle$ ). In particular, with $\left\{X_{i}\right\}$ as in (2.1) and $\left\{h_{i}\right\}$ any orthonormal basis for $\mathfrak{a}$, we have

$$
\Omega=\sum_{i=1}^{\ell} h_{i}^{2}+\sum_{i=1}^{m} X_{i}^{2} .
$$

Remarks. The essential data for constructing $\mathfrak{b}$ and $\Omega$ consist of the restriction of the inner product to $\mathfrak{a} \times \mathfrak{a}$ and the set $\left\{\alpha_{i}\right\}$ of roots. Indeed, given this information we can construct $\mathfrak{b}$ using the commutation relations (2.1), and define an inner product on $\mathfrak{b}$ extending the given inner product on $\mathfrak{a}$ by making $\left\{X_{i}\right\}$ orthonormal. Note that $\left(\mathbb{R}^{\times}\right)^{m}$ acts on $\mathfrak{b}$ as automorphisms, via $t \cdot H=H$, for $H \in \mathfrak{a}$, and $t \cdot X_{i}=t_{i} X_{i}$, where $t=\left(t_{1}, \ldots, t_{m}\right)$. Relative to this action, all the inner products on $\mathfrak{b}$ constructed this way are equivalent, and the corresponding Laplacians are likewise transformed.

In this section we shall study elements of $U(\mathfrak{b})$ in terms of their commutation properties with $\Omega$, with the goal of determining the structure of the commutant $U(\mathfrak{b})^{\Omega}$. For this, fix the bases $\left\{h_{i}\right\}$ and $\left\{X_{i}\right\}$ as above. If $\alpha \in \mathfrak{a}^{*}$ then $H_{\alpha} \in \mathfrak{a}$ is defined by

$$
\left\langle H_{\alpha}, H\right\rangle=\alpha(H), \quad H \in \mathfrak{a} .
$$

We note that the present set-up admits the following inductive scheme:

For any subset $P \subseteq\{1, \ldots, m\}$, let $\mathfrak{u}_{P}=\operatorname{span}\left\{X_{i} \mid i \notin P\right\}$, and set $\mathfrak{b}_{P}=\mathfrak{a} \oplus \mathfrak{u}_{P}$, with the inner product on $\mathfrak{b}_{P}$ being the restriction of $\langle\cdot, \cdot\rangle$. The orthogonal projection

$$
\mu_{P}: \mathfrak{b} \rightarrow \mathfrak{b}_{P}
$$

is a Lie algebra homomorphism, which we extend to a homomorphism of enveloping algebras. Then $\Omega_{P}=\mu_{P}(\Omega)$ is the Laplacian on $\mathfrak{b}_{P}$. (Note that the subscript $P$ will consistently mean that the indices $i \in P$ are to be omitted.)

If $P, Q$ are subsets of $\{1, \ldots, m\}$, then the homomorphisms $\mu_{P}, \mu_{Q}$ are coherent, in the sense that

$$
\mu_{P}\left(\mu_{Q}(Z)\right)=\mu_{P \cup Q}(Z), \quad Z \in U(\mathfrak{b}) .
$$

When $P=\{1, \ldots, m\}$, then we write $\mu_{P}=\mu$, so that $\mu: U(\mathfrak{b}) \rightarrow U(\mathfrak{a})$. When $P=\{j\}$, we write $\mu_{P}=\mu_{j}$.

We now turn to some explicit calculations of commutation relations with $\Omega$.

Lemma 2.1. Suppose $Z \in U(\mathfrak{u}), \alpha \in \mathfrak{a}^{*}$, and $[H, Z]=\alpha(H) Z$ for all $H \in \mathfrak{a}$. Then $[\Omega, Z]=Z\left(2 H_{\alpha}+\langle\alpha, \alpha\rangle\right)$. 
Proof. Since $Z \in U(\mathfrak{u})$, one has

$$
\begin{aligned}
{[\Omega, Z] } & =\sum\left[h_{i}^{2}, Z\right]=\sum \alpha\left(h_{i}\right)\left(Z h_{i}+h_{i} Z\right) \\
& =Z\left\{\sum 2 \alpha\left(h_{i}\right) h_{i}+\alpha\left(h_{i}\right)^{2}\right\} .
\end{aligned}
$$

But $\left\langle h_{i}, h_{j}\right\rangle=\delta_{i j}$, so that $\sum \alpha\left(h_{i}\right) h_{i}=H_{\alpha}$ and $\sum \alpha\left(h_{i}\right)^{2}=\langle\alpha, \alpha\rangle$.

Lemma 2.2. Let $u \in U(\mathfrak{b})$, and suppose that

$$
[\Omega, u]+h u+c u=0,
$$

where $c \in \mathbb{R}$ and $h \in \mathfrak{a}$. Assume that for all $n_{i} \in \mathbb{N}$,

Then $u=0$.

$$
h+2 \sum_{i=1}^{m} n_{i} H_{\alpha_{i}} \neq 0 \text {. }
$$

Proof. By induction on $\operatorname{dim}(\mathfrak{u})$. When $\operatorname{dim}(\mathfrak{u})=0$, condition (2.3) becomes $(h+c) u=0$, with the hypothesis $h \neq 0$. Thus $u=0$ in this case.

Now assume the lemma is true when $\operatorname{dim}(\mathfrak{u}) \leqq m-1$, and consider the case $\operatorname{dim}(\mathfrak{u})=m$. Applying the homomorphism $\mu_{m}$ to (2.3), we obtain by the induction hypothesis that $\mu_{m}(u)=0$. Hence $u=X_{m} u_{1}$ for some $u_{1} \in U(\mathfrak{b})$. By Lemma 2.1

$$
\begin{aligned}
{\left[\Omega, X_{m} u_{1}\right] } & =\left[\Omega, X_{m}\right] u_{1}+X_{m}\left[\Omega, u_{1}\right] \\
& =X_{m}\left\{2 H_{\alpha_{m}} u_{1}+\left\langle\alpha_{m}, \alpha_{m}\right\rangle u_{1}+\left[\Omega, u_{1}\right]\right\} .
\end{aligned}
$$

But by (2.3) one also has

$$
\begin{aligned}
{\left[\Omega, X_{m} u_{1}\right] } & =-h X_{m} u_{1}-c X_{m} u_{1} \\
& =-X_{m}\left\{h u_{1}+\left(c+\alpha_{m}(h)\right) u_{1}\right\}
\end{aligned}
$$

Hence $u_{1}$ satisfies

$$
\left[\Omega, u_{1}\right]+h_{1} u_{1}+c_{1} u_{1}=0,
$$

where $h_{1}=h+2 H_{\alpha_{m}}$ and $c_{1}=c+\alpha_{m}(h)+\left\langle\alpha_{m}, \alpha_{m}\right\rangle$. Since $h_{1}$ satisfies the same hypothesis as $h$, we may repeat this argument, to obtain elements $u_{k} \in U(\mathfrak{b})$ such that $u=X_{m}^{k} u_{k}$ for $k=1,2,3, \ldots$. Hence $u=0$.

Lemma 2.3. Let $u \in U(\mathfrak{b})$ satisfy

$$
[\Omega, u]+h u+c u=0
$$

for some $h \in \mathfrak{a}$ and $c \in \mathbb{R}$. Assume that for some $j \leqq m$ and all $n_{i} \in \mathbb{N}, h+2 \sum_{i \neq j} n_{i} H_{\alpha_{i}} \neq 0$. Then $u \in X_{j} U(\mathfrak{b})$.

Proof. Apply Lemma 2.2 to $\mu_{j}(u)$ to conclude that $u \in \operatorname{Ker}\left(\mu_{j}\right)=X_{j} U(\mathfrak{b})$.

Let $\sigma_{i}: \mathfrak{b} \rightarrow \mathfrak{b}$ be the orthogonal reflection defined by $\sigma_{i}(H)=H$ for $H \in \mathfrak{a}$ and

$$
\sigma_{i}\left(X_{j}\right)=(-1)^{\delta_{i j}} X_{j}, \quad 1 \leqq i, j \leqq m .
$$

Then $\sigma_{i}$ is clearly an automorphism of $\mathfrak{b}$, and so extends to an automorphism of $U(\mathfrak{b})$. Define

$$
{ }^{\circ} U(\mathfrak{b})=\left\{b \in U(\mathfrak{b}): \sigma_{i}(b)=b, \quad 1 \leqq i \leqq m\right\} .
$$


In terms of a PBW basis $\left\{X^{I} h^{J}: I \in \mathbb{N}^{m}, J \in \mathbb{N}^{\ell}\right\}$ for $U(\mathfrak{b}),{ }^{\circ} U(\mathfrak{b})$ is the subspace spanned by $\left\{X^{I} h^{J}: I \in(2 \mathbb{N})^{m}, J \in \mathbb{N}^{\ell}\right\}$.

Here $X^{I}=X_{1}^{i_{1}} \ldots X_{m}^{i_{m}}$ and $h^{J}=h_{1}^{j_{1}} \ldots h_{\ell}^{j_{\ell}}$.

Lemma 2.4. Let $P \subset\{1, \ldots, m\}, u \in U(\mathfrak{b})$, and suppose $\mu_{k}(u)=0$ for $k \in P$. Then $u=\left(\prod_{k \in P} X_{k}\right) v$ for some $v \in U(\mathfrak{b})$. If also $u \in{ }^{\circ} U(\mathfrak{b})$, then $u=\left(\prod_{k \in P} X_{k}^{2}\right) v^{\prime}$ for some $v^{\prime} \in{ }^{\circ} U(\mathfrak{b})$.

Proof. Use the PBW basis $\left\{X^{I} h^{J}\right\}$ for $U(\mathfrak{b})$ and the commutativity of $\left\{X_{i}\right\}$.

Theorem 2.5. Assume that the set of roots $\left\{\alpha_{1}, \ldots, \alpha_{m}\right\}$ is linearly independent. Then

(1) $\mu: U(\mathfrak{b})^{\Omega} \rightarrow U(\mathfrak{a})$ is injective;

(2) $\operatorname{deg} \mu(T)=\operatorname{deg} T$, for $T \in U(\mathfrak{b})^{\Omega}$;

(3) $U(\mathfrak{b})^{\Omega}$ is commutative;

(4) $U(\mathfrak{b})^{\Omega} C^{\circ} U(\mathfrak{b})$.

Proof. The independence of the roots implies that there is a basis $\left\{H_{j}\right\}$ for a such that $\alpha_{i}\left(H_{j}\right)=\delta_{i j}$. One has $\left[H_{i}, X_{j}\right]=\delta_{i j} X_{j}$ and

$$
\Omega=\sum a_{i j} H_{i} H_{j}+\sum X_{i}^{2}
$$

where $a_{i j}=\left\langle\alpha_{i}, \alpha_{j}\right\rangle$.

Fix the basis $\left\{H_{i}\right\}$ and define linear maps $\Delta_{i}: U(\mathfrak{a}) \rightarrow U(\mathfrak{a})$ by

$$
\Delta_{i} f\left(H_{1}, \ldots, H_{\ell}\right)=f\left(H_{1}, \ldots, H_{i}+2, \ldots, H_{\ell}\right)-f\left(H_{1}, \ldots, H_{i}, \ldots, H_{\ell}\right),
$$

for $1 \leqq i \leqq \ell$, where $f$ is any polynomial in $\ell$ variables. One verifies by induction on $\operatorname{deg}(u)$ that

$$
\left[u, X_{i}^{2}\right]=X_{i}^{2} \Delta_{i} u, \quad u \in U(\mathfrak{a})
$$

Also one has

$$
\left[H_{i} H_{j}, X^{K}\right]=X^{K}\left(k_{i} H_{j}+k_{j} H_{i}+k_{i} k_{j}\right),
$$

for any multi-exponent $K=\left(k_{1}, \ldots, k_{m}\right) \in \mathbb{N}^{m}$.

Suppose $T \in U(\mathfrak{b})$. Then $T$ may be written uniquely as a finite sum

$$
T=\sum_{K} X^{K} u_{K}, u_{K} \in U(\mathfrak{a}) .
$$

By (2.4) and (2.5) the equation $[\Omega, T]=0$ is equivalent to

$$
\sum_{K} X^{K} u_{K} g_{K}=\sum_{i, K} X_{i}^{2} X^{K} \Delta_{i} u_{K}
$$

where

$$
g_{K}=\sum_{i, j} a_{i j}\left(2 k_{i} H_{j}+k_{i} k_{j}\right) .
$$

If we introduce the notation

$$
\alpha_{K}=\sum_{i} k_{i} \alpha_{i}, \quad \text { for } \quad K=\left(k_{1}, \ldots, k_{m}\right),
$$


then $\alpha_{K} \neq 0$ for $K \neq 0$, and we have

$$
g_{K}=2 H_{\alpha_{K}}+\left\langle\alpha_{K}, \alpha_{K}\right\rangle \text {. }
$$

In particular, $\operatorname{deg}\left(g_{K}\right)=1$ for any $K \neq 0$, and Eq. (2.6) is equivalent to the following recursion for $\left\{u_{K}\right\}$ :

$$
u_{K} g_{K}=\sum_{i=1}^{m} \Delta_{i} u_{K-2 \varepsilon_{i}}
$$

for $K \neq 0$. Here $\varepsilon_{i}=(0, \ldots, 1, \ldots, 0)\left(1\right.$ in $i^{\text {th }}$ place $)$, and we set $u_{J}=0$ if any coordinate of $J$ is negative.

The assertions of the theorem now follow from (2.7). Note that $\operatorname{deg}\left(\Delta_{i} u\right) \leqq \operatorname{deg}(u)-1$, while $\operatorname{deg}\left(u g_{K}\right)=\operatorname{deg} u+1$, if $u \neq 0$. Hence by induction we see that $\operatorname{deg} u_{K} \leqq \operatorname{deg} u_{0}-|K|$, so that $\operatorname{deg} T=\operatorname{deg} u_{0}$. But $u_{0}=\mu(T)$, which proves (1) and (2). Since $\mu$ is a homomorphism, (3) follows from (1), and (4) is clear inductively from (2.7). Q.E.D.

Given $\alpha \in \mathfrak{a}^{*}, \alpha \neq 0$, denote by $s_{\alpha}: \mathfrak{a} \rightarrow \mathfrak{a}$ the orthogonal reflection through the hyperplane $\alpha=0$; thus

$$
s_{\alpha}(H)=H-(2 \alpha(H) /\langle\alpha, \alpha\rangle) H_{\alpha} .
$$

Extend $s_{\alpha}$ to an automorphism of $U(\mathfrak{a})$.

Theorem 2.6. Assume that $\alpha \neq 0$ is a root of a on $\mathfrak{u}$. If $v \in U(\mathfrak{b})^{\Omega}$ then $s_{\alpha} \mu(v)=\mu(v)$.

Proof. Assume $\alpha=\alpha_{i}$, and let $P$ be the set $\{1, \ldots, m\}$ with $i$ omitted. Then $\mu(v)=\mu\left(\mu_{P}(v)\right)$, and $\operatorname{dim}\left(\mathfrak{u}_{P}\right)=1$. Thus it suffices to treat the case $\operatorname{dim} \mathfrak{u}=1$, since $\mu_{P}(v) \in U\left(\mathfrak{b}_{P}\right)^{\Omega_{P}}$.

We now assume $\operatorname{dim} u=1$. Since $\alpha \neq 0$, we may assume that the orthonormal basis $\left\{h_{j}\right\}$ for $\mathfrak{a}$ is taken such that $\alpha\left(h_{j}\right)=0$ for $j \geqq 2$. Set $\mathfrak{a}_{1}=\operatorname{span}\left\{h_{2}, \ldots, h_{\ell}\right\}$, and write $\mathscr{K}=U\left(\mathfrak{a}_{1}\right)$. Then $\left[X_{1}, \mathscr{K}\right]=0,\left.s_{\alpha}\right|_{\mathscr{K}}=1$, and $U(\mathfrak{a})=\mathscr{K}\left[h_{1}\right]$. We shall prove that

$$
U(\mathfrak{b})^{\Omega}=\mathscr{K}[\Omega] .
$$

This will yield the theorem, since

$$
s_{\alpha}\left(h_{1}\right)=-h_{1} \quad \text { and } \quad \mu(\Omega)=h_{1}^{2} \bmod \mathscr{K} .
$$

To prove (2.8), write $H=h_{1}, X=X_{1}, c=\alpha\left(h_{1}\right)$, and note that the only non-trivial commutation relation in $\mathfrak{b}$ is

$$
[H, X]=c X .
$$

Define $\Delta f(H)=f(H+2 c)-f(H)$, if $f \in \mathscr{K}[H]$. Let $T \in U(\mathfrak{b})^{\Omega}$, and write, by Theorem 2.5,

$$
T=\sum_{k \leqq d} X^{2 k} f_{k}(H),
$$

where $f_{k}(H) \in \mathscr{K}[H]$. Equation (2.7) can be written in this case as

$$
4 k f_{k}(H)(c H+k\langle\alpha, \alpha\rangle)=\Delta f_{k-1}(H) .
$$


In particular, for $k=d+1$, one has $\Delta f_{d}(H)=0$. Since $c \neq 0$ and $f_{d}$ is a polynomial, this implies that $f_{d}(H)=u_{0} \in \mathscr{K}$. But then $T-u_{0} \Omega^{d} \in U(\mathfrak{b})^{\Omega}$ and has degree in $X$ less than $d$. Thus (2.8) follows by induction on $d$.

\section{Inversion of the Symbol Map}

Motivated by the example of the "periodic Toda lattice" (Sect. 5), for which the roots are not independent, we next construct a (partial) right inverse to the symbol map

$$
\mu: U(\mathfrak{b})^{\Omega} \rightarrow U(\mathfrak{a})
$$

under the following hypotheses on the set of roots $\pi=\left\{\alpha_{1}, \ldots, \alpha_{m}\right\} \subset a^{*}$ :

$$
\left\{\begin{array}{l}
\text { For all } i, \alpha_{i} \neq 0, \text { and } \\
\pi \sim\left\{\alpha_{i}\right\} \text { is linearly independent. }
\end{array}\right.
$$

Let $W \subset$ Aut $(\mathfrak{a})$ be the group generated by the reflections $\left\{s_{\alpha_{i}} ; 1 \leqq i \leqq m\right\}$. (Write $W=W_{\pi}$ if necessary to indicate the dependence on $\pi$.) By Theorem 2.6, $\mu\left(U(\mathfrak{b})^{\Omega}\right) \subseteq U(\mathfrak{a})^{W}$. For any non-empty subset $P \subset\{1, \ldots, m\}$, condition (3.1) and Theorem 2.5 give the injectivity of

$$
\mu: U\left(\mathrm{~b}_{P}\right)^{\Omega_{P}} \rightarrow U(\mathfrak{a})^{W} .
$$

Define

$$
U(\mathfrak{a})_{0}^{W}=\bigcap_{|P| \geqq 1} \mu\left(U\left(\mathfrak{b}_{P}\right)^{\Omega_{P}}\right) .
$$

Then given $u \in U(\mathfrak{a})_{0}^{W}$ and a non-empty subset $P \subset\{1, \ldots, m\}$, there is a unique element $w_{P}(u) \in U\left(\mathfrak{b}_{P}\right)^{\Omega_{P}}$ such that

$$
\mu\left(w_{P}(u)\right)=u .
$$

Furthermore, $\operatorname{deg} w_{P}(u)=\operatorname{deg} u$ and $w_{P}(u) \in^{\circ} U(\mathfrak{b})$. Via the natural inclusion $U\left(\mathfrak{b}_{p}\right) \subset U(\mathfrak{b})$, we may view the map $u \mapsto w_{p}(u)$ as an injective degree-preserving algebra homomorphism

$$
w_{P}: U(\mathfrak{a})_{0}^{W} \rightarrow{ }^{\circ} U(\mathfrak{b}) .
$$

Now define a linear map $w^{\prime}: U(\mathfrak{a})_{0}^{W} \rightarrow{ }^{\circ} U(\mathfrak{b})$ by

$$
w^{\prime}(u)=\sum(-1)^{|P|+1} w_{P}(u)
$$

(sum over all non-empty subsets $P$ of $\{1, \ldots, m\}$ ). This map gives a "first approximation" to the desired right inverse for $\mu$; under suitable conditions on $\pi$ and $\operatorname{deg}(u)$ we will show that $w^{\prime}(u) \in U(\mathfrak{b})^{\Omega}$.

To obtain some basic properties of the map $w^{\prime}$, recall that for every subset $P \subseteq\{1, \ldots, m\}$ there is a homomorphism

$$
\mu_{P}: U(\mathfrak{b}) \rightarrow U\left(\mathfrak{b}_{P}\right) .
$$

Lemma 3.1. If $|P| \geqq 1$ and $u \in U(\mathfrak{a})_{0}^{W}$, then

$$
\mu_{P}\left(w^{\prime}(u)\right)=w_{P}(u) .
$$


Proof. Suppose $P=\{i\}$ and $\emptyset \neq Q \subseteq\{1, \ldots, m\}$. By the injectivity of $\mu$ on $U\left(\mathfrak{b}_{P}\right)^{\Omega_{P}}$, one has

$$
\mu_{i}\left(w_{Q}(u)\right)= \begin{cases}w_{Q \cup\{i\}}(u), & \text { if } \quad i \notin Q \\ w_{Q}(u), & \text { if } \quad i \in Q .\end{cases}
$$

Hence by (3.2),

$$
\mu_{i}\left(w^{\prime}(u)\right)=\sum_{i \in Q}(-1)^{|Q|+1} w_{Q}(u)+\sum_{i \notin Q}(-1)^{|Q|+1} w_{Q \cup\{i\}}(u)
$$

(sum over non-empty subsets $Q$ of $\{1, \ldots, m\}$ ). Writing $Q=\{i\} \cup Q_{0}$, with $i \notin Q_{0}$, in the first summand in (3.5), one obtains cancellation with a corresponding term in the second summand, except for the term with $Q=\{i\}$. This proves (3.4) when $|P|=1$.

Suppose now $P=Q \cup\{i\}$, where $i \notin Q$. Then $\mu_{P}=\mu_{Q} \mu_{i}$, so $\mu_{P}\left(w^{\prime}(u)\right)=\mu_{Q}\left(w_{\{i\}}(u)\right)$. But $\mu \circ \mu_{Q}=\mu$, so $w_{P}(u)$ and $\mu_{P}\left(w^{\prime}(u)\right)$ both project onto $u$. Hence they are equal, by Theorem 2.5, Q.E.D.

Next we measure the extent to which $w^{\prime}(u)$ fails to commute with $\Omega$.

Lemma 3.2. There is a linear map $w^{\prime \prime}: U(\mathfrak{a})_{0}^{W} \rightarrow{ }^{\circ} U(\mathfrak{b})$ such that

$$
\left[\Omega, w^{\prime}(u)\right]=X_{1}^{2} \ldots X_{m}^{2} w^{\prime \prime}(u) \text {. }
$$

Furthermore, $\operatorname{deg} w^{\prime \prime}(u) \leqq \operatorname{deg}(u)+1-2 m$, so that $w^{\prime \prime}(u)=0$ if $\operatorname{deg}(u) \leqq 2 m-2$.

Proof. For $1 \leqq i \leqq m$, one has $\mu_{i}(\Omega)=\Omega_{i}$. Hence by Lemma 3.1,

$$
\mu_{i}\left(\left[\Omega, w^{\prime}(u)\right]\right)=\left[\Omega_{i}, w_{i}(u)\right]=0 .
$$

Since $w^{\prime}(u), \Omega \in{ }^{\circ} U(\mathfrak{b})$ and ${ }^{\circ} U(\mathfrak{b})$ is a subalgebra, we have $\left[\Omega, w^{\prime}(u)\right] \in{ }^{\circ} U(\mathfrak{b})$, so (3.6) follows from Lemma 2.4. Since $\operatorname{deg} w^{\prime}(u) \leqq \operatorname{deg}(u)$, the estimate for $\operatorname{deg} w^{\prime \prime}(u)$ is immediate.

Lemma 3.3. Let $u \in U(\mathfrak{a})_{0}^{W}, R \in U(\mathfrak{b})$, and set

$$
T=w^{\prime}(u)+X_{1}^{2} \ldots X_{m}^{2} R .
$$

Then $T$ commutes with $\Omega$ iff $R$ satisfies

$$
[\Omega, R]+4\left(H_{\gamma}+\langle\gamma, \gamma\rangle\right) R+w^{\prime \prime}(u)=0,
$$

where $\gamma=\alpha_{1}+\ldots+\alpha_{m}$.

Proof. By Lemma 2.1, for any $R \in U(\mathrm{~b})$,

$$
\left[\Omega, X_{1}^{2} \ldots X_{m}^{2} R\right]=X_{1}^{2} \ldots X_{m}^{2}\left(4 H_{\gamma}+4\langle\gamma, \gamma\rangle\right) R+X_{1}^{2} \ldots X_{m}^{2}[\Omega, R] .
$$

Thus by (3.6) it is clear that $[T, \Omega]=0$ is equivalent to (3.8).

Theorem 3.4. Assume the set of roots $\left\{\alpha_{1} \ldots \alpha_{m}\right\}$ is linearly independent, and let $u \in U(\mathfrak{a})_{0}^{W}$ be given. Then there exists $T \in U(\mathfrak{b})^{\Omega}$ such that $\mu(T)=u$ iff $T=w^{\prime}(u)+X_{1}^{2} \ldots X_{m}^{2} R$, where $R \in{ }^{\circ} U(\mathfrak{b})$ and satisfies equation (3.8).

Remark. Since $H_{\gamma}$ satisfies the hypotheses of Lemma 2.3 (for $h$ ) when $\left\{\alpha_{i}\right\}$ is independent, a solution $R$ to (3.8) is uniquely determined by $u$. 
Proof of Theorem 3.4. The map

$$
\mu: U(\mathfrak{b})^{\Omega} \rightarrow U(\mathfrak{a})^{W}
$$

is injective, by Theorem 2.5. Suppose there exists $T \in U(\mathfrak{b})^{\Omega}$ with $\mu(T)=u$. Then $T \in{ }^{\circ} U(\mathfrak{b})$ and

$$
\mu_{i}\left(T-w^{\prime}(u)\right)=0, \quad 1 \leqq i \leqq m,
$$

by Lemma 3.1. But $w^{\prime}(u) \in{ }^{\circ} U(\mathfrak{b})$, so that $T-w^{\prime}(u)=X_{1}^{2} \ldots X_{m}^{2} R$ for some $R \in{ }^{\circ} U(\mathfrak{b})$, by Lemma 2.4. Now apply Lemma 3.3 to complete the proof.

We now turn to the situation that arises in the periodic Toda lattice and its generalizations. Namely, we assume that $\operatorname{dim} \mathfrak{a}=\ell, \operatorname{dim}(\mathfrak{u})=\ell+1$, and that the relation among the roots is of the form

$$
\alpha_{\ell+1}+\sum_{i=1}^{\ell} n_{i} \alpha_{i}=0
$$

where $n_{i} \geqq 1$ are integers. Set

$$
\xi=X_{1}^{n_{1}} \ldots X_{\ell}^{n_{\ell}} X_{\ell+1} .
$$

Note that $[H, \xi]=0$, so $\xi$ is in the center of $U(\mathfrak{b})$.

Lemma 3.5. Let $T \in U(\mathfrak{b})^{\Omega}$ and suppose $\mu(T)=0$. Then $T \in \xi U(\mathfrak{b})^{\Omega}$.

Proof. For $1 \leqq i \leqq \ell+1$ one has

$$
\mu_{i}(T) \in U\left(\mathfrak{b}_{i}\right)^{\Omega_{2}}
$$

But $\mu\left(\mu_{i}(T)\right)=\mu(T)=0$, so by condition (3.1) and Theorem $2.5, \mu_{i}(T)=0$. Hence $T=X_{1} \ldots X_{\ell+1} v$ for some $v \in U(\mathfrak{b})$, by Lemma 2.4 .

If all $n_{i}=1$, we are done. If not, assume the roots $\alpha_{i}$ are numbered so that $n_{1} \geqq n_{2} \geqq \ldots \geqq n_{\ell}$. Set

$$
\gamma=\sum_{i=1}^{\ell+1} \alpha_{i}=\sum_{i=1}^{\ell}\left(1-n_{i}\right) \alpha_{i}
$$

Then by Lemma 2.1,

$$
0=[\Omega, T]=X_{1} \ldots X_{\ell+1}\left\{\left(2 H_{\gamma}+\langle\gamma, \gamma\rangle\right) v+[\Omega, v]\right\},
$$

which gives $\left(2 H_{\gamma}+\langle\gamma, \gamma\rangle\right) v+[\Omega, v]=0$.

But $n_{1}>1$ and $\left\{\alpha_{i}: 1 \leqq i \leqq \ell\right\}$ is linearly independent, so that $\gamma \notin \operatorname{span}\left\{\alpha_{j} ; j \neq 1\right\}$. Hence by Lemma 2.2, $v=X_{1} v^{\prime}$. We may repeat this argument until we get $v=X_{1}^{n_{1}} v_{1}$. Now use (2.5) again, to get

$$
0=[\Omega, T]=X_{1}^{n_{1}} X_{2} \ldots X_{\ell+1}\left\{\left(2 H_{\beta}+\langle\beta, \beta\rangle\right) v_{1}+\left[\Omega, v_{1}\right]\right\},
$$

where $\beta=\sum_{i=2}^{\ell}\left(1-n_{i}\right) \alpha_{i}$. If $n_{2}>1$, then $\beta \notin \operatorname{span}\left\{\alpha_{j} ; j \neq 2\right\}$, so by Lemma 2.2 , $v_{1}=X_{2} v_{1}^{\prime}$. Repeat to get $v_{1}=X_{2}^{n_{2}} v_{2}$, etc. Finally, we have $T=\xi v$. Now $[T, \Omega]=0$ implies $[v, \Omega]=0$, so that $T \in \xi U(\mathfrak{b})^{\Omega}$. 
Lemma 3.6. Let $\sigma=\sigma_{\ell+1} \in \operatorname{Aut}(\mathfrak{b})$ be the reflection sending $X_{\ell+1}$ to $-X_{\ell+1}(c f$. Sect. 2). Suppose $T \in U(\mathfrak{b})^{\Omega}$.

(i) If $\sigma(T)=-T$, then $T \in \xi U(\mathfrak{b})^{\Omega}$.

(ii) If $\mu(T)=0$ and $\sigma(T)=T$, then $T \in \xi^{2} U(\mathfrak{b})^{\Omega}$.

Proof. (i) Since $\mu \circ \sigma=$ identity, we have $T \in \operatorname{Ker} \mu$, so Lemma 3.5 applies.

(ii) By Lemma 3.5, $T=\xi T^{\prime}$ for some $T^{\prime} \in U(\mathfrak{b})^{\Omega}$. Since $\sigma(\xi)=-\xi$, one has $\sigma\left(T^{\prime}\right)=-T^{\prime}$, so $T^{\prime}=\xi T^{\prime \prime}$ by (i).

Theorem 3.7. Assume that $\operatorname{dim}(\mathfrak{u})=\ell+1$, and that the roots $\left\{\alpha_{1}, \ldots, \alpha_{\ell+1}\right\}$ satisfy (3.1) and (3.9). Define $\xi$ by (3.10).

(a) If $U(\mathfrak{a})_{0}^{W}$ is generated by elements $u_{1}, \ldots, u_{n}$ of degrees $\leqq 2 \ell$, then $U(\mathfrak{b})^{\Omega}$ is generated by $\xi$ and $w^{\prime}\left(u_{1}\right), \ldots, w^{\prime}\left(u_{n}\right)$.

(b) If $2 \operatorname{deg} \xi \geqq \max _{i \neq j}\left(\operatorname{deg} u_{i}+\operatorname{deg} u_{j}\right)$, with $\left\{u_{i}\right\}$ as in (a), then $U(\mathrm{~b})^{\Omega}$ is commutative.

Proof. (a) Since $m=\ell+1$ in Lemma 3.2, we have $w^{\prime \prime}\left(u_{i}\right)=0$, so that $w^{\prime}\left(u_{i}\right) \in U(\mathfrak{b})^{\Omega}$. Given $T \in U(\mathfrak{b})^{\Omega}$, we can thus construct $T^{\prime}$ in the algebra generated by $\left\{w^{\prime}\left(u_{i}\right)\right\}$ with $\mu\left(T^{\prime}\right)=\mu(T)$ and $\operatorname{deg} T \geqq \operatorname{deg} T^{\prime} \quad$ [since $\mu(T)$ is a polynomial in $\left\{u_{i}\right\}$, and $\left.\operatorname{deg} w^{\prime}\left(u_{i}\right)=\operatorname{deg}\left(u_{i}\right)\right]$. Hence $T=T^{\prime}+\xi v$ for some $v \in U(\mathfrak{b})^{\Omega}$, by Lemma 3.5. Since $\operatorname{deg}(v)<\operatorname{deg} T$, we obtain the result by induction on $\operatorname{deg}(T)$.

(b) Note that $\left[w^{\prime}\left(u_{i}\right), w^{\prime}\left(u_{j}\right)\right] \in \operatorname{Ker}(\mu) \cap^{\circ} U(\mathfrak{b})^{\Omega}$, and has degree at most $r-1$, where $r=\max _{i \neq j}\left(\operatorname{deg}\left(u_{i}\right)+\operatorname{deg}\left(u_{j}\right)\right)$. But this commutator is divisible by $\xi^{2}$, by Lemma 3.6(ii), and $\operatorname{deg} \xi^{2} \geqq r$, by assumption. Hence $w^{\prime}\left(u_{i}\right)$ and $w^{\prime}\left(u_{j}\right)$ must commute. Since $\xi$ is central in $U(\mathfrak{b})$, part (b) follows from (a) in this case.

\section{Structure of $U(b)^{\Omega}$ for Dynkin Diagrams}

We shall say that a subset $\pi=\left\{\alpha_{1}, \ldots, \alpha_{\ell}\right\} \subset \mathfrak{a}^{*}$ defines a Dynkin diagram if it is a set of simple positive roots for a reduced root system $R_{\pi} \subset a^{*}$. This is equivalent to the following conditions :

$\left(D_{\mathrm{I}}\right) \pi$ is a basis for $\mathrm{a}^{*}$;

$\left(D_{\mathrm{II}}\right)$ the group $W$ generated by the reflections $\left\{s_{\alpha_{i}}\right\}$ is finite;

$\left(D_{\text {III }}\right)$ the numbers $a_{i j}=2\left\langle\alpha_{i}, \alpha_{j}\right\rangle /\left\langle\alpha_{j}, \alpha_{j}\right\rangle$ are integers $\leqq 0$, for $i \neq j$.

(For these and other properties of root systems cited below, Bourbaki [6].) The matrix $\left[a_{i j}\right]$ is called the Cartan matrix of $\pi$.

Theorem 4.1. Let $\mathfrak{b}=\mathfrak{a} \oplus \mathfrak{u}$ as in Sect. 2. Assume that $\operatorname{dim} \mathfrak{a}=\operatorname{dim} \mathfrak{u}$ and the set $\pi$ of roots of a on $\mathfrak{u}$ defines a Dynkin diagram. Then

$$
\mu: U(\mathfrak{b})^{\Omega} \rightarrow U(\mathfrak{a})^{W}
$$

is an algebra isomorphism.

Remark. The condition that $\pi$ define a Dynkin diagram depends on the inner product on $\mathfrak{a}^{*}$, dual to the given inner product on $\mathfrak{a}$. Given any base $\pi \subset \mathfrak{a}^{*}$ defining a Dynkin diagram, we can construct $\mathfrak{b}$, an inner product $\langle\cdot, \cdot\rangle$, and Laplacian $\Omega$, as remarked in Sect. 2. 
Corollary 4.2. Let $u_{1}, \ldots, u_{\ell}$ be a set of homogeneous, algebraically independent generators for $U(\mathfrak{a})^{W}$. Then there are unique elements $T_{1}, \ldots, T_{\ell} \in U(\mathfrak{b})^{\Omega}$ such that $\mu\left(T_{j}\right)=u_{j}$ and

$$
U(\mathfrak{b})^{\Omega}=\mathbb{R}\left[T_{1}, \ldots, T_{\ell}\right] .
$$

Furthermore, if $\operatorname{deg}\left(u_{j}\right) \leqq 2 \ell-2$, then $T_{j}=w^{\prime}\left(u_{j}\right)$, where the map $w^{\prime}$ is defined by (3.3).

Remarks. 1. For the irreducible classical Dynkin diagrams of type $A_{\ell}$ or $D_{\ell}, \ell \geqq 3$, the basic invariants $\left\{u_{j}\right\}$ all have degrees $\leqq 2 \ell-2$. Also, starting with a diagram of type $A_{\ell}$ or $D_{\ell}$ and applying the inductive construction of Sect. 3 for the map $w^{\prime}$, one obtains subdiagrams which are direct sums of types $A_{r}$ and/or $D_{r}$. Thus all the elements $T_{j}$ can be constructed inductively via $w^{\prime}$ in cases $A_{\ell}$ and $D_{\ell}$. [For $A_{2}$, it follows easily by Theorem 3.4 that $w^{\prime}\left(u_{2}\right) \in U(\mathfrak{b})^{\Omega}$, where $u_{2}$ is a basic invariant of order 3.]

2. For the diagrams of type $B_{\ell}$ or $C_{\ell}$, all but one basic invariant have degrees $\leqq 2 \ell-2$, so Corollary 4.2 applies to these. For an invariant $u_{\ell}$ of degree $2 \ell$, we note that in Lemma 3.2, $\operatorname{deg} w^{\prime \prime}\left(u_{\ell}\right) \leqq 1$. Using Theorem 3.4, we find that $T_{\ell}=\mu^{-1}\left(u_{\ell}\right)$ is given by the formula

$$
T_{\ell}=w^{\prime}\left(u_{\ell}\right)+\frac{\varepsilon\left(w^{\prime \prime}\left(u_{\ell}\right)\right)}{4\langle\gamma, \gamma\rangle} X_{1}^{2} \ldots X_{\ell}^{2},
$$

where $\gamma=\alpha_{1}+\ldots+\alpha_{\ell}$ and

$$
\varepsilon: U(\mathfrak{b}) \rightarrow \mathbb{R}
$$

is the augmentation homomorphism $[\varepsilon(T)=$ constant term of $T]$. Thus for all diagrams of classical type there is an explicit inductive algorithm to determine $T_{1}, \ldots, T_{\ell}$ from $u_{1}, \ldots, u_{\ell}$.

Proof of Theorem 4.1. There exists a real semi-simple Lie algebra $\mathfrak{g}$, with Iwasawa decomposition $\mathfrak{g}=\mathfrak{n}+\mathfrak{a}+\mathfrak{l}$, as in Sect. 1 , such that $\pi$ is the set of simple roots for the action of $\operatorname{ad}(\mathfrak{a})$ on $\mathfrak{n}$, and $\langle\cdot, \cdot\rangle$ restricted to $\mathfrak{a} \times \mathfrak{a}$ is the Cartan-Killing form. Thus $\mathfrak{b} \cong \mathfrak{a} \oplus(\mathfrak{n} /[\mathfrak{n}, \mathfrak{n}])$, and as previously noted we may assume that the inner product on $\mathfrak{b}$ is consistent with formula (1.4), since rescaling the inner product on $\mathfrak{u}$ can be done via an automorphism of $\mathfrak{b}$ which is trivial on $\mathfrak{a}$.

With these normalizations we may apply Proposition 1.4 to conclude that

$$
\Omega=\tilde{\gamma}(C)+\langle\varrho, \varrho\rangle,
$$

where $C$ is the Casimir operator in $U(\mathfrak{g})$ (notation as in Sect. 1). Thus

$$
\tilde{\gamma}\left(U(\mathfrak{g})^{\mathfrak{f}}\right) \subseteq U(\mathfrak{b})^{\Omega},
$$

and it follows by Lemma 1.2 and Theorem 2.6 that

$$
\mu: U(\mathfrak{b})^{\Omega} \rightarrow U(\mathfrak{a})^{W}
$$

is surjective. The injectivity of $\mu$ is immediate from the linear independence of the roots and Theorem 2.5 .

Proof of Corollary 4.2. The existence of the generators $u_{1}, \ldots, u_{\ell}$ follows from Chevalley's theorem. Applying Theorem 4.1 to the subalgebras $\mathfrak{b}_{P}$, we conclude 
that

$$
U(\mathfrak{a})_{0}^{W}=U(\mathfrak{a})^{W}
$$

[Eq. (3.2)]. Now apply Theorem 3.7, noting that $n=\ell$ in this case.

\section{Structure of $U(b)^{\Omega}$ for Extended Dynkin Diagrams}

Assume that $\pi=\left\{\alpha_{1}, \ldots, \alpha_{\ell}\right\}$ defines a Dynkin diagram, in the sense of Sect. 4. Let $R=R_{\pi}$ be the associated reduced root system. We shall assume throughout this section that $R$ is irreducible. Let $\beta \in R$, and write $\alpha_{\ell+1}=-\beta$.

Definition 5.1. The set $\pi_{\beta}=\left\{\alpha_{1}, \ldots, \alpha_{\ell+1}\right\}$ defines an extended Dynkin diagram if every subset obtained by deleting one element from $\pi_{\beta}$ gives a Dynkin diagram.

Proposition 5.1. The set $\pi_{\beta}$ defines an extended Dynkin diagram iff $\beta$ is dominant:

$$
\left\langle\beta, \alpha_{i}\right\rangle \geqq 0 \quad \text { for } 1 \leqq i \leqq \ell .
$$

When (5.1) holds, then

$$
\beta=\sum_{i=1}^{\ell} n_{i} \alpha_{i}, \quad \text { with } \quad n_{i} \geqq 1 \quad \text { for all } i .
$$

Proof. We first prove that (5.1) implies (5.2). Let $\left\{\omega_{1}, \ldots, \omega_{\ell}\right\}$ be the fundamental weights relative to $\pi$, defined by $\left\langle\alpha_{i}, \omega_{j}\right\rangle=(1 / 2) \delta_{i j}\left\langle\alpha_{i}, \alpha_{i}\right\rangle$. Then by (5.1), $\beta=\sum m_{i} \omega_{i}$ with $m_{i} \geqq 0$. But one also has $\omega_{i}=\sum c_{i j} \alpha_{j}$ with $c_{i j} \geqq 0$. Hence the coefficients $n_{i}$ in (5.2) are non-negative. Set $J=\left\{j: n_{j}=0\right\}$. Since $\left\langle\alpha_{i}, \alpha_{j}\right\rangle \leqq 0$ for $i \neq j$, we have $\sum_{i} n_{i}\left\langle\alpha_{i}, \alpha_{j}\right\rangle \leqq 0$ for all $j \in J$. But by (5.1), this forces $\left\langle\alpha_{i}, \alpha_{j}\right\rangle=0$ when $i \notin J, j \in J$. The irreducibility of $R$ then implies that $J$ is empty, proving (5.2).

Suppose now $\beta \in R$. If $\pi_{\beta}$ defines an extended Dynkin diagram, then by condition $\mathrm{D}_{\text {III }}$ of Sect. $4,\left\langle\beta, \alpha_{i}\right\rangle=-\left\langle\alpha_{\ell+1}, \alpha_{i}\right\rangle \geqq 0$ for $1 \leqq i \leqq \ell$. Conversely, suppose (5.1) holds. Then from (5.2) we see that any proper subset of $\pi_{\beta}$ is linearly independent. Since $\beta \in R$, the reflection $s_{\beta}$ is in the Weyl group of $\pi$, and $2\left\langle\beta, \alpha_{i}\right\rangle /\left\langle\alpha_{i}, \alpha_{i}\right\rangle$ is a non-negative integer. Hence conditions $\left(\mathrm{D}_{\mathrm{I}}\right)-\left(\mathrm{D}_{\mathrm{III}}\right)$ of Sect. 4 hold for the sets $\pi_{\beta} \sim\left\{\alpha_{i}\right\}, 1 \leqq i \leqq \ell$. This proves that $\pi_{\beta}$ is an extended Dynkin diagram in the sense of Definition 5.1.

Example. Let $\beta=\tilde{\alpha}$ be the largest root of the system $R$, relative to the ordering given by the base $\pi$. Then $\beta$ satisfies condition (5.1). The set $\pi_{\beta}$ is the completed Dynkin diagram corresponding to the root system $R$, in Bourbaki's terminology.

The classification of extended Dynkin diagrams follows easily from the classification of Dynkin diagrams, Proposition 5.1, and the following properties of root systems :

(A) $R$ has elements of at most two lengths.

(B) The group $W$ operates transitively on the roots of a given length, and for $\alpha \in R$, the orbit $W \cdot \alpha$ contains exactly one dominant root.

For the diagrams $A_{\ell}, D_{\ell}, E_{6}, E_{7}, E_{8}$, for which all roots have the same length, the only choice for $\beta$ is the largest root $\tilde{\alpha}$. When $R$ has roots of two lengths, there is 
also a unique short dominant root $\beta$. Hence the diagrams $B_{\ell}, C_{\ell}, F_{4}, G_{2}$ each give rise to two extended Dynkin diagrams (see Fig. 5.1).

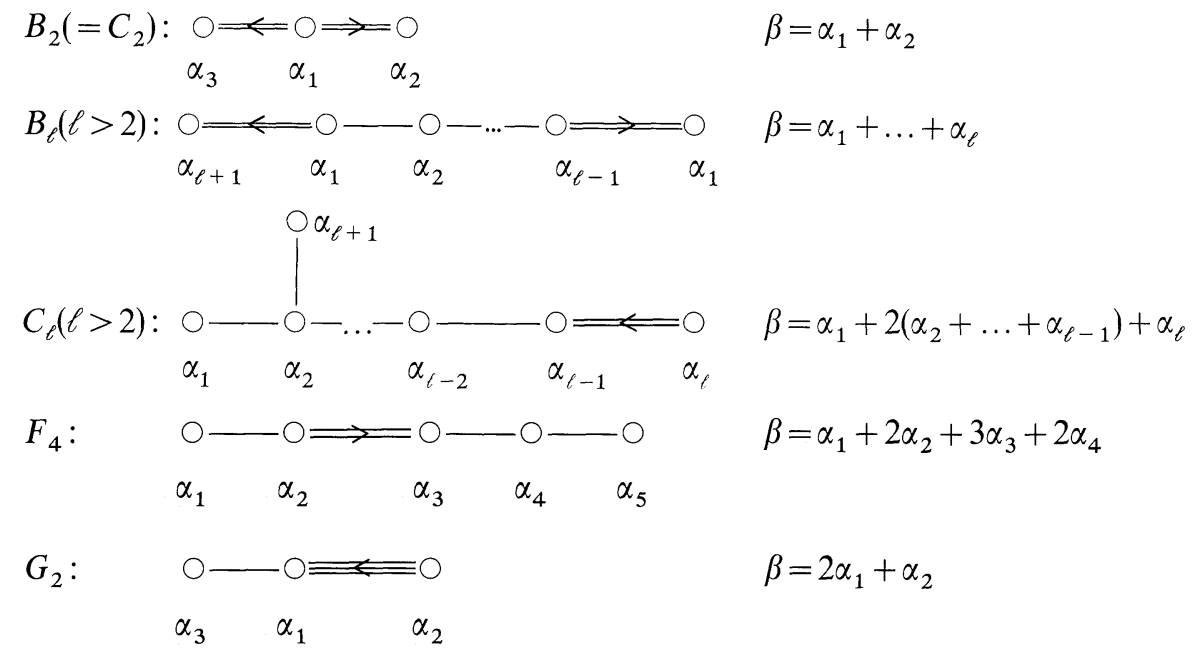

Fig. 5.1. Extended Dynkin diagrams determined by $\beta=$ short dominant root $\left(\alpha_{\ell+1}=-\beta\right)$

Let $\pi_{\beta}$ be an extended Dynkin diagram, and form the Lie algebra $\mathfrak{b}=\mathfrak{a} \oplus \mathfrak{u}$ as in Sect. 2, with $\left\{\alpha_{1}, \ldots, \alpha_{\ell+1}\right\}$ the roots of $\operatorname{ad}(\mathfrak{a})$ on $\mathfrak{u}$, and the inner product $\left.\langle\cdot, \cdot\rangle\right|_{\mathfrak{a} \times \mathfrak{a}}$ being the one associated with the root system [see remarks after formula (2.2)]. Let $\Omega$ be the Laplace operator for $\mathfrak{b}$. We are thus in the situation of Sect. 3 , since

$$
\alpha_{\ell+1}+\sum_{i=1}^{\ell} n_{i} \alpha_{i}=0,
$$

with $\left\{n_{i}\right\}$ as in (5.2). Set $|\beta|=\sum_{i=1}^{\ell} n_{i}$, so that the element $\xi \in U(\mathfrak{b})$ defined by (3.10) has degree $|\beta|+1$. Let $\left\{u_{1}, \ldots, u_{\ell}\right\} \subset U(\mathfrak{a})^{W}$ be a set of homogeneous, algebraically independent generators.

Theorem 5.2. Assume that $\pi$ is a Dynkin diagram of classical type or $E_{6}$, and that $\mathfrak{b}$ and $\Omega$ are defined from the extended Dynkin diagram $\pi_{\beta}$. Then

(a) $U(\mathfrak{b})^{\Omega}$ is generated by $\xi$ and $w^{\prime}\left(u_{1}\right), \ldots, w^{\prime}\left(u_{\ell}\right)$;

(b) $U(\mathfrak{b})^{\Omega}$ is commutative if $\beta=\tilde{\alpha}$ is the largest positive root, or if $\pi=C_{\ell}$ and $\beta=$ short dominant root.

Remark. For the diagram $B_{\ell}$ with $\beta$ the short dominant root, we prove a weaker version of (b) in Sect. 6.

Proof. We use Theorem 3.7. The diagrams $A_{\ell}, B_{\ell}, C_{\ell}, D_{\ell}$, and $E_{6}$ are exactly those for which all basic invariants in $U(\mathfrak{a})^{W}$ have degree $\leqq 2 \ell$. By Theorem 4.1 we know that $U(\mathfrak{a})_{0}^{W}=U(\mathfrak{a})^{W}$, so part (a) follows from Theorem 3.7.

For (b), we note that when $\beta=\tilde{\alpha}$, then $\operatorname{deg} \xi=|\beta|+1=h$, the Coxeter number of $W$. But one also has $h=\max \left(\operatorname{deg}\left(u_{i}\right)\right)$, for any Weyl group. Thus part (b) of 
Theorem 3.7 applies. When $\pi$ is the diagram $C_{\ell}$, and $\beta$ is the short dominant root, then $\beta=\varepsilon_{1}+\varepsilon_{2}=\alpha_{1}+2\left(\alpha_{2}+\ldots+\alpha_{\ell-1}\right)+\alpha_{\ell}$, in the notation of Bourbaki (loc. cit.), so that $|\beta|=2 \ell-2$. But $\operatorname{deg} u_{i}=2 i$, so $\max _{i \neq j}\left(\operatorname{deg} u_{i}+\operatorname{deg} u_{j}\right)=4 \ell-2=2 \operatorname{deg} \xi$. Again part (b) of Theorem 3.7 applies.

\section{Centralizer of the Laplacian in the Poisson Algebra}

If $\mathfrak{g}$ is a Lie algebra (over a field of characteristic zero), recall $[12,18]$ that the symmetric tensor algebra $S(\mathrm{~g})$ carries the structure of a Poisson algebra; there is a Lie algebra multiplication $\{f, g\}$ on $S(\mathfrak{g})$ such that

(i) $\{X, Y\}=[X, Y]$, for $X, Y \in \mathfrak{g}$;

(ii) $\{f g, h\}=\{f, h\} g+f\{g, h\}$ for $f, g, h \in S(\mathfrak{g})$.

The bracket operation $\{f, g\}$ can be defined via $U(\mathfrak{g})$ as follows (in Sect. 7 we give an equivalent differential-geometric definition): Let $\left\{U_{n}(g)\right\}_{n \geqq 0}$ be the canonical filtration on $U(\mathfrak{g})$, and let $S^{n}(\mathfrak{g})$ be the subspace of $S(\mathfrak{g})$ of homogeneous elements of degree $n$. Let

$$
\begin{aligned}
j: U(\mathfrak{g}) & \rightarrow S(\mathfrak{g}) \\
j_{n}: U_{n}(\mathfrak{g}) / U_{n-1}(\mathfrak{g}) & \rightarrow S^{n}(\mathfrak{g})
\end{aligned}
$$

be the canonical isomorphisms given by the Poincaré-Birkhoff-Witt theorem. If $P \in U_{n}(\mathfrak{g})$ and $Q \in U_{m}(\mathfrak{g})$, then one defines

$$
\left\{j_{n}(P), j_{m}(Q)\right\}=j_{m+n-1}(P Q-P Q) .
$$

(Since $\left[U_{m}, U_{n}\right] \subseteq U_{m+n-1}$, it is clear that the right side of (6.1) depends only on $P$ $\bmod U_{n-1}$ and $Q \bmod U_{m-1}$.) Property (i) of $\{\cdot, \cdot\}$ is immediate, and (ii) is easily verified. Note that

$$
\left\{S^{m}(\mathfrak{g}), S^{n}(\mathfrak{g})\right\} \cong S^{m+n-1}(\mathfrak{g})
$$

The elements $f, g \in S(\mathfrak{g})$ will be said to Poisson-commute if $\{f, g\}=0$. If $f=j(P)$ and $g=j(Q)$, with $f, g$ homogeneous of degrees $m, n$ respectively, then the Poissoncommutativity of $f, g$ is equivalent to the condition $[P, Q] \in U_{m+n-2}(\mathfrak{g})$. In particular, if $P$ and $Q$ commute in $U(\mathfrak{g})$, then $j(P)$ and $j(Q)$ Poisson-commute in $S(\mathfrak{g})$.

We return to the general context of Sect. 2. Let $\Omega$ be the Laplacian defined there, and define

$$
S(\mathfrak{b})^{j(\Omega)}=\{f \in S(\mathfrak{b}):\{f, j(\Omega)\}=0\} .
$$

Since $j(\Omega) \in S^{2}(\mathfrak{b})$, we see by (6.2) that $S(\mathfrak{b})^{j(\Omega)}$ is a graded Poisson subalgebra of $S(\mathfrak{b})$. Also

$$
j: U(\mathfrak{b})^{\Omega} \rightarrow S(\mathfrak{b})^{j(\Omega)}
$$

is an injective linear map. Corresponding to the decomposition $\mathfrak{b}=\mathfrak{a} \oplus \mathfrak{n}$ there is a projection map $v: S(\mathfrak{b}) \rightarrow S(\mathfrak{a})$, and of course $j: S(\mathfrak{a}) \cong U(\mathfrak{a})$. One checks that the 
diagram

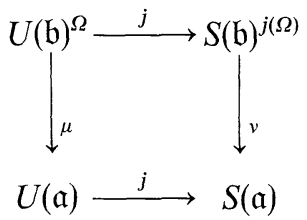

is commutative.

In this section, we adapt the arguments of Sect. 2 to study the Poissoncommutant $S(\mathfrak{b})^{j(\Omega)}$. We show that it is Lie-isomorphic to the enveloping algebra commutant $U(\mathfrak{b})^{\Omega}$, when $\mathfrak{b}$ and $\Omega$ are defined by Dynkin diagrams or certain extended Dynkin diagrams.

Define

$$
{ }^{\circ} S(\mathfrak{b})=j\left({ }^{\circ} U(\mathfrak{b})\right),
$$

so that the elements of ${ }^{\circ} S(\mathfrak{b})$ only contain even powers of the basis $\left\{X_{i}\right\}$ for $\mathfrak{u}$. We then have the following Poisson-algebra analogue of Theorem 2.5:

Theorem 6.1. Assume that the set of roots $\left\{\alpha_{1}, \ldots, \alpha_{m}\right\}$ is linearly independent. Then

(1) $v: S(\mathfrak{b})^{j(\Omega)} \rightarrow S(\mathfrak{a})$ is injective;

(2) $\operatorname{deg} v(Q)=\operatorname{deg} Q$, for $Q \in S(\mathfrak{b})^{j(\Omega)}$;

(3) $S(\mathfrak{b})^{j(\Omega)}$ is Poisson-commutative;

(4) $S(\mathfrak{b})^{j(\Omega)} C^{\circ} S(\mathfrak{b})$.

Proof. Since $S(\mathfrak{b})^{j(\Omega)}$ is graded, it suffices to consider only homogeneous elements in $S(\mathfrak{b})$; for these we can calculate Poisson brackets using (6.1). This amounts to taking only the top-order terms in the calculations of Sect. 2. We sketch the resulting modifications in the proof of Theorem 2.5 to obtain Theorem 6.1.

Fix the basis $\left\{H_{i}\right\}$ for $\mathfrak{a}$ as in that proof. Then for a homogeneous polynomial $f$ of degree $m$,

$$
\Delta_{i} f\left(H_{1}, \ldots, H_{\ell}\right) \equiv 2 \frac{\partial}{\partial H_{i}} f\left(H_{1}, \ldots, H_{\ell}\right)
$$

$\bmod U_{m-1}(\mathfrak{a})$. Thus if $T \in U_{m}(\mathfrak{b})$ is given by (2.5), then by (2.6) the Poisson commutativity equation $[\Omega, T] \equiv 0 \bmod U_{m}(\mathfrak{b})$ is equivalent to

$$
\sum_{K} X^{K} u_{K} H_{\alpha_{K}} \equiv \sum_{i, K} X_{i}^{2} X^{K} \frac{\partial}{\partial H_{i}}\left(u_{K}\right)
$$

$\bmod U_{m}(\mathfrak{b})$. It follows that the analogue of Eq. (2.7) for Poisson commutativity of a homogeneous element $Q=\sum X^{K} u_{K} \in S^{m}(\mathfrak{b})$ is

$$
H_{\alpha_{\mathrm{K}}} u_{K}=\sum \frac{\partial}{\partial H_{i}} u_{K^{-2 \varepsilon_{i}}} \text {. }
$$

[Here $u_{K} \in S^{m-|K|}(\mathfrak{a})$ and the product $X^{K} u_{K}$ is in $S(\mathfrak{b})$.]

Assertions (1), (2), and (4) of Theorem 6.1 now follow from (6.5), just as the analogous statements in Theorem 2.5 followed from Eq. (2.7). For Poisson- 
commutativity, note that

$$
\{S(\mathfrak{b}), S(\mathfrak{b})\} \cong \mathfrak{u} S(\mathfrak{b}),
$$

so the map $v$ annihilates all Poisson brackets. Hence the injectivity of $v$ on $S(\mathfrak{b})^{j(\Omega)}$ implies assertion (3), finishing the proof.

Theorem 6.2. Assume that $\alpha \neq 0$ is a root of $\mathfrak{a}$ on $\mathfrak{u}$. If $Q \in S(\mathfrak{b})^{j(\Omega)}$, then $s_{\alpha} v(Q)=v(Q)$.

Proof. The same reduction to the case $\operatorname{dim} u=1$ as in the proof of Theorem 2.6 applies here. Let the notation be as in that proof, and suppose that

$$
T=\sum_{k \leqq d} X^{2 k} f_{k}(H) \in U_{2 d}(\mathfrak{b}),
$$

where $f_{k}(H)$ is a polynomial of degree $\leqq 2(d-k)$ in $H$ with coefficients in $\mathscr{K}$. Note that Eq. (2.9) can be written as

$$
2 k H f_{k}(H) \equiv(d / d H) f_{k-1}(H),
$$

$\bmod U_{2 d-1}(\mathfrak{b})$. Now transfer Eq. (6.6) to $S(\mathfrak{b})$ via the map $j$, and argue by induction on $d$ that $S(\mathfrak{b})^{j(\Omega)}=\mathscr{K}[j(\Omega)]$, as in the proof of Theorem 2.6. [Here we identify $\mathscr{K}=U\left(\mathfrak{a}_{1}\right)$ with $S\left(\mathfrak{a}_{1}\right) \subset S(\mathfrak{b})$.] We leave the details to the reader.

Corollary 6.3. Assume that $\operatorname{dim}(\mathfrak{u})=\operatorname{dim}(\mathfrak{a})=\ell$ and the roots $\left\{\alpha_{1}, \ldots, \alpha_{\ell}\right\}$ define $a$ Dynkin diagram. Then $S(\mathfrak{b})^{j(\Omega)}$ is Poisson-commutative, and

is a linear isomorphism.

$$
j: U(\mathfrak{b})^{\Omega} \rightarrow S(\mathfrak{b})^{j(\Omega)}
$$

Proof. By Theorem 6.2, v:S(b) $)^{j(\Omega)} \rightarrow S(\mathfrak{a})^{W}$, where $W$ is the Weyl group of the Dynkin diagram. But by the commutativity of diagram (6.3) and Theorems 4.1 and 6.1, the map $v$ is a linear isomorphism. Hence (6.7) is also bijective, Q.E.D.

Now we assume that $\pi=\left\{\alpha_{1}, \ldots, \alpha_{\ell}\right\}$ is a reduced, irreducible Dynkin diagram, and we form an extended Dynkin diagram as in Sect. 5. We then have the following Poisson algebra analogue of Theorem 5.2:

Theorem 6.4. Assume that $\pi$ is a Dynkin diagram of classical type or $E_{6}$, and that $\mathfrak{b}$ and $\Omega$ are defined from an extended Dynkin diagram $\pi_{\beta}$. Then

(a) the canonical map $j: U(\mathfrak{b})^{\Omega} \rightarrow S(\mathfrak{b})^{j(\Omega)}$ is bijective;

(b) $S(\mathfrak{b})^{j(\Omega)}$ is Poisson-commutative.

Remark. Part (b) is immediate from (a) whenever $U(\mathfrak{b})^{\Omega}$ is known to be commutative. In the case of the diagram $B_{\ell}$ with $\beta$ the short dominant root, which could not be treated by the methods of Sect. 5, we do not know if $U(\mathfrak{b})^{\Omega}$ is commutative. Nonetheless, the Poisson-commutativity (b) does hold.

Proof. (a) First note that an obvious modification of the proof of Lemma 3.5 shows that

$$
\operatorname{Ker}(v)=j(\xi) S(\mathfrak{b}),
$$

where $\xi$ is defined by (3.10), with the exponents $\left\{n_{i}\right\}$ being the coefficients of $\beta$ in terms of $\left\{\alpha_{1}, \ldots, \alpha_{\ell}\right\}$. 
Next, given $Q \in S^{m}(\mathfrak{b})^{j(\Omega)}$, we know by Theorem 6.2 that $v(Q) \in S(\mathfrak{a})^{W}$, where $W=W_{\pi}$. Hence by Theorem 5.2 there is $T \in U_{m}(\mathfrak{b})^{\Omega}$ such that $j \circ \mu(T)=v(Q)$. Hence by (6.3), $Q-j(T) \in \operatorname{Ker}(v)$. Thus $Q-j(T)=j(\xi) Q_{1}$, where $Q_{1}$ Poisson-commutes with $j(\Omega)$ and has degree less than $m$. By induction on $m$ this proves (a).

(b) By Theorem 5.2 and part (a), the only case to be checked is $\pi=\beta_{\ell}, \beta=$ short dominant root. In the notation of $[6], \beta=\varepsilon_{1}=\alpha_{1}+\ldots+\alpha_{\ell}$, so that $n_{i}=1$ and $\operatorname{deg} \xi=\ell+1$ in this case. By part (a) of Theorem 5.2, it will suffice to show that the elements $w^{\prime}\left(u_{i}\right)$ Poisson-commute in $U(\mathfrak{b})$. But one has $\operatorname{deg} w^{\prime}\left(u_{i}\right)=\operatorname{deg} u_{i}=2 i$, and by Lemma 3.6,

$$
\left[w^{\prime}\left(u_{i}\right), w^{\prime}\left(u_{j}\right)\right]=\xi^{2} v_{i j}
$$

where $v_{i j} \in U(\mathfrak{b})^{\Omega}$. Since $\operatorname{deg}\left(v_{i j}\right) \leqq 2(i+j-\ell)-3<\operatorname{deg} \xi$, we know that $v_{i j}$ is a polynomial in $\left\{w^{\prime}\left(u_{k}\right)\right\}$, by part (a) of Theorem 5.2. In particular, $\operatorname{deg}\left(v_{i j}\right)$ is even. Hence the left side of (6.9) has degree at most $2 i+2 j-2$, establishing Poissoncommutativity.

\section{Cotangent Bundles and Coadjoint Orbits}

In order to relate the enveloping algebra results of the previous sections to the Hamiltonian systems of "Toda Lattice" type, we now recall some basic symplectic geometry. We will use standard differential-geometric notation, following [20] (except for occasional minus signs arising from a choice between right and left actions). Since the results are mostly known $[4,12,14]$, the proofs will be generally sketched or left to the reader.

Recall that if $M$ is a smooth manifold and $T^{*}(M)$ is the cotangent bundle of $M$, then $T^{*}(M)$ has a natural symplectic structure, obtained as follows:

Let $\pi: T^{*}(M) \rightarrow M$ be the projection. If $\xi \in T^{*}(M)$ and $v \in T\left(T^{*}(M)_{\xi}\right)$, define $\theta_{\xi}(v)=\xi\left(d \pi_{\xi}(v)\right)$. Set $\omega=-d \theta$. Then $\omega$ is a closed non-singular 2-form on $T^{*}(M)$. If $\phi \in C_{\mathbb{R}}^{\infty}\left(T^{*}(M)\right)$, let the Hamiltonian vector field $v_{\phi}$ on $T^{*}(M)$ be defined by $\omega\left(v_{\phi}, u\right)=u(\phi)$ for all smooth vector fields $u$ on $T^{*}(M)$. The Poisson bracket of $\phi, \psi \in C_{\mathbb{R}}^{\infty}\left(T^{*}(M)\right)$ is then defined as

$$
\{\phi, \psi\}=v_{\phi}(\psi)=\omega\left(v_{\psi}, v_{\phi}\right) .
$$

We now specialize to the case $M=G$, a real Lie group. Let $g$ denote the Lie algebra of $G$, which we view as the space of left-invariant real vector fields on $G$. For $X \in \mathfrak{g}$ and $g \in G$, one has $X_{g} \in T(G)_{g}$ given by

$$
X_{g}(\phi)=\left.\frac{d}{d t} \phi(g \exp t X)\right|_{t=0},
$$

for $\phi \in C^{\infty}(G)$.

We can trivialize the cotangent bundle of $G$ by defining $\psi: G \times \mathfrak{g}^{*} \rightarrow T^{*}(G)$ as

$$
\psi(g, f)\left(X_{g}\right)=f(X),
$$

for $g \in G, f \in \mathfrak{g}^{*}$, and $X \in \mathfrak{g}$. Then $\psi$ is a vector bundle isomorphism. The left action of $G$ on $T^{*}(G)$ becomes the action $s \cdot(g, f)=(s g, f)$ on $G \times \mathrm{g}^{*}$ under this isomorphism. The right action of $G$ on $T^{*}(G)$ becomes

$$
(g, f) \cdot s=\left(g s, s^{-1} \cdot f\right),
$$


where $s \cdot f(X)=f\left(\operatorname{Ad}(s)^{-1} X\right)$ for $s \in G, f \in \mathfrak{g}^{*}, X \in \mathfrak{g}$, with Ad the adjoint representation of $G$. Thus the left $G$-invariant functions on $T^{*}(G)$ correspond under $\psi$ to the functions on $\mathrm{g}^{*}$.

If $\lambda \in \mathfrak{g}^{*}, f \in \mathfrak{g}^{*}$, we define $\lambda_{f} \in T\left(\mathfrak{g}^{*}\right)_{f}$ to be the directional derivative in $\mathfrak{g}^{*}$ at $f$ in the direction $\lambda$. If $v \in T\left(G \times \mathfrak{g}^{*}\right)_{(g, f)}$, then there are elements $X(v) \in \mathfrak{g}$ and $\lambda(v) \in \mathfrak{g}^{*}$ such that

$$
v=\left(X(v)_{g}, \lambda(v)_{f}\right)
$$

If $\theta$ is the canonical 1 -form on $T^{*}(G)$, one has $\psi^{*}(\theta)_{\xi}(v)=f(X(v))$, where $\xi=(g, f)$. Using the standard formula $d \theta(A, B)=A \theta(B)-B \theta(A)-\theta([A, B])$, for $A, B$ vector fields, one calculates the symplectic structure on $G \times \mathrm{g}^{*}$ as follows:

Lemma 7.1. For $(g, f) \in G \times \mathrm{g}^{*}$ and $v, w \in T\left(G \times \mathrm{g}^{*}\right)_{(g, f)}$, one has

$$
\left(\psi^{*} \omega\right)_{(g, f)}(v, w)=f([X(v), X(w)])-\lambda(v)(X(w))+\lambda(w)(X(v)) .
$$

We abuse the notation and henceforth use the symbol $\omega$ to denote $\psi^{*} \omega$ on $G \times \mathrm{g}^{*}$.

Let $H \in C_{\mathbb{R}}^{\infty}\left(\mathfrak{g}^{*}\right)$. Using the canonical identification between $\left(\mathfrak{g}^{*}\right)^{*}$ and $\mathfrak{g}$, we may consider the differential of $H$ as a function from $\mathfrak{g}^{*}$ to $\mathfrak{g}$. Thus for $f, \lambda \in \mathfrak{g}^{*}$, we have

$$
\lambda(d H(f))=\left.\frac{d}{d t} H(f+t \lambda)\right|_{t=0} .
$$

We also look upon $H$ as a function on $G \times g^{*}$, by setting $H(g, f)=H(f)$, for $g \in G, f \in \mathfrak{g}^{*}$. Let $v_{H}$ be the corresponding Hamiltonian vector field on $G \times \mathfrak{g}^{*}$. The next lemma is proved by the obvious calculation, using (7.3), and is left to the reader. [For $X, Y \in \mathfrak{g}, f \in \mathfrak{g}^{*}$, write $(X \cdot f)(Y)=-f([X, Y])$.]

Lemma 7.2. If $H \in C_{\mathbb{R}}^{\infty}\left(\mathfrak{g}^{*}\right), g \in G, f \in \mathfrak{g}^{*}$, then

$$
\left(v_{H}\right)_{(g, f)}=\left(d H(f)_{g},-(d H(f) \cdot f)_{f}\right) .
$$

Furthermore, if $H_{1}, H_{2} \in C_{\mathbb{R}}^{\infty}\left(g^{*}\right)$, then

$$
\left\{H_{1}, H_{2}\right\}_{(g, f)}=f\left(\left[d H_{1}(f), d H_{2}(f)\right]\right) .
$$

In particular, if $X \in \mathfrak{g}$, then $X$ defines a function $H_{X}$ on $\mathfrak{g}^{*}$ by $H_{X}(\lambda)=\lambda(X)$. The corresponding Hamiltonian vector field is

$$
\left(v_{H_{X}}\right)_{(g, f)}=\left(X_{g},-(X \cdot f)_{f}\right) .
$$

Comparing (7.6) and (7.2), we see that $v_{H_{X}}$ is in fact the vector field on $G \times \mathrm{g}^{*}$ corresponding to $X$ under the differential of the right action of $G$. By (7.5) we have

$$
\left\{H_{X}, H_{Y}\right\}=H_{[X, Y]},
$$

for $X, Y \in \mathfrak{g}$, so the right $G$-action on $T^{*}(G)$ is Hamiltonian [4]. Furthermore, the map $X \rightarrow H_{X}$ extends to an algebra isomorphism between $S(\mathfrak{g})$ and the real-valued polynomial functions on $\mathfrak{g}^{*}$, and (7.7) shows that the Poisson bracket on $S(\mathfrak{g})$ defined via $U(\mathfrak{g})$ in Sect. 6 agrees with the Poisson bracket coming from $T^{*}(G)$ (cf. [12]).

Given $f \in \mathfrak{g}^{*}$, let $\mathcal{O}=\mathcal{O}^{f}=G \cdot f$ be the coadjoint orbit of $f$, and $G_{f}$ the stabilizer of $f$. If $X \in \mathfrak{g}$, then $(X \cdot f)_{f} \in T(\mathcal{O})_{f}$, and we let $\omega^{\mathscr{O}}$ be the symplectic form on $\mathcal{O}$ such 
that

$$
\omega_{f}^{\mathcal{O}}\left((X \cdot f)_{f},(Y \cdot f)_{f}\right)=f([Y, X])
$$

for $X, Y \in \mathfrak{g}$. If $H \in C_{\mathbb{R}}^{\infty}\left(\mathfrak{g}^{*}\right)$, let $H^{\mathcal{O}}=\left.H\right|_{\mathscr{O}}$, and denote by $X_{H}^{\mathcal{O}}$ the Hamiltonian vector field on $\mathcal{O}$ corresponding to $H^{\mathcal{O}}$ via $\omega^{\mathcal{O}}$.

Lemma 7.3. One has

$$
\left(X_{H}^{\mathfrak{O}}\right)_{f}=-(d H(f) \cdot f)_{f}
$$

If $H_{1}, H_{2} \in C_{\mathbb{R}}^{\infty}\left(\mathfrak{g}^{*}\right)$, then

$$
\left\{H_{1}^{\mathcal{O}}, H_{2}^{\mathcal{O}}\right\}=\left\{H_{1}, H_{2}\right\}^{\mathcal{O}}
$$

Proof. Let $Y \in \mathfrak{g}$. Then

$$
\omega_{f}^{\mathcal{O}}\left(X_{H}, Y \cdot f\right)=\left.\frac{d}{d t} H(f+t Y \cdot f)\right|_{t=0}=f([d H(f), Y]) .
$$

Comparing with (7.8), we get formula (7.9). By (7.1) and (7.9), we have

$$
\begin{aligned}
\left\{H_{1}, H_{2}\right\}(f) & =\left.\frac{d}{d t} H_{2}\left(f-t d H_{1}(f) \cdot f\right)\right|_{t=0} \\
& =-\left(d H_{1}(f) \cdot f\right)\left(d H_{2}(f)\right) \\
& =f\left(\left[d H_{1}(f), d H_{2}(f)\right]\right) .
\end{aligned}
$$

Thus (7.10) follows from (7.5)

Remark. Let $\tau: G \times \mathcal{O} \rightarrow \mathcal{O}$ be the projection. Then

$$
\left(\tau^{\mathfrak{O}}\right)^{*}\left(\omega^{\mathfrak{O}}\right)=\left.\omega\right|_{G \times \mathcal{O}},
$$

as one calculates easily using Lemma 7.1. Also for $H \in C_{\mathbb{R}}^{\infty}\left(\mathfrak{g}^{*}\right)$, one has

$$
\tau_{*}^{\mathfrak{O}}\left(v_{H}\right)=X_{H}^{\mathfrak{O}},
$$

so the Hamiltonian flow on $\mathcal{O}$ generated by $H^{\mathcal{O}}$ is the projection onto $\mathcal{O}$ of the flow on $T^{*}(G)$ generated by $H$.

We now turn to the case $\mathfrak{g}=\mathfrak{b}=\mathfrak{a} \oplus \mathfrak{u}$, as in Sect. 2 , with $\left\{\alpha_{1}, \ldots, \alpha_{m}\right\} \subset \mathfrak{a}^{*}$ the roots of $\mathfrak{a}$ on $\mathfrak{u}$ and $\left\{X_{1}, \ldots, X_{m}\right\} \subset \mathfrak{u}$ an orthonormal basis of root vectors $\left(\left[H, X_{i}\right]=\alpha_{i}(H) X_{i}\right.$ for $\left.H \in \mathfrak{a}\right)$. It is immediate that

$$
\text { Center }(\mathfrak{b})=\mathfrak{a}_{0} \oplus \mathfrak{u}_{0},
$$

where

$$
\mathfrak{a}_{0}=\bigcap_{i=1}^{m} \operatorname{Ker}\left(\alpha_{i}\right), \mathfrak{u}_{0}=\operatorname{span}\left\{X_{i}: \alpha_{i}=0\right\} .
$$

Thus replacing $\mathfrak{b}$ by $\mathfrak{b} /\left(\mathfrak{a}_{0} \oplus \mathfrak{u}_{0}\right)$, we shall assume that:

$$
\alpha_{i} \neq 0,1 \leqq i \leqq m, \text { and }\left\{\alpha_{1}, \ldots, \alpha_{m}\right\} \text { spans } a^{*} .
$$

Let $\left\{X_{1}^{*}, \ldots, X_{m}^{*}\right\} \subset \mathfrak{b}^{*}$ be the dual basis to $\left\{X_{1}, \ldots, X_{m}\right\}$, extended to be zero on $\mathfrak{a}$, and consider $\left\{\alpha_{1}, \ldots, \alpha_{m}\right\}$ as elements of $\mathfrak{b}^{*}$ which are zero on $\mathfrak{u}$. One easily 
calculates that the coadjoint action of $\mathfrak{b}$ on $\mathfrak{b}^{*}$ is given by

$$
\begin{aligned}
& H \cdot X_{j}^{*}=-\alpha_{j}(H) X_{j}^{*}, H \cdot \alpha_{j}=0, \\
& X_{i} \cdot X_{j}^{*}=\delta_{i j} \alpha_{j}, X_{i} \cdot \alpha_{j}=0 .
\end{aligned}
$$

Let $B$ be the connected and simply-connected Lie group with Lie algebra $\mathfrak{b}$. The map

$$
(H, X) \mapsto \exp (H) \exp (X)
$$

is a global diffeomorphism from $\mathfrak{a} \times \mathfrak{u}$ onto $B$. Suppose $f=\sum c_{i} X_{i}^{*}$. Then by (7.12),

$$
\exp (H) \exp (X) \cdot f=\sum_{i=1}^{m} c_{i} e^{-\alpha_{\imath}(H)} X_{i}^{*}+\sum_{i=1}^{m} c_{i} X_{i}^{*}(X) \alpha_{i}
$$

Assume that $f$ is generic, i.e. $\operatorname{dim} \mathcal{O}^{f}$ is maximal. By (7.11) and (7.13) one has $c_{i} \neq 0$ for all $i$ in this case, and the isotopy group

$$
\begin{aligned}
B_{f} & =\left\{\exp (X): X \in \mathfrak{u} \text { and } \sum c_{i} X_{i}^{*}(X) \alpha_{i}=0\right\} \\
& =\{\exp (X):(H \cdot f)(X)=0, \text { all } H \in \mathfrak{a}\} .
\end{aligned}
$$

By (7.11), $\operatorname{dim} \mathfrak{a} \cdot f=\operatorname{dim} \mathfrak{a}$, so we see that $\operatorname{dim} B_{f}=\operatorname{dim} u-\operatorname{dim} a$. Thus $\operatorname{dim} \mathcal{O}^{f}=\operatorname{dim} B-\operatorname{dim} B_{f}=2 \operatorname{dim} \mathfrak{a}$.

We shall assume the $\alpha_{i}$ are numbered so that $\left\{\alpha_{1}, \ldots, \alpha_{\ell}\right\}$ is linearly independent $(\ell=\operatorname{dim} \mathfrak{a})$. Define

$$
\mathfrak{u}_{1}=\operatorname{span}\left\{X_{1}, \ldots, X_{\ell}\right\}, \mathfrak{b}_{1}=\mathfrak{a} \oplus \mathfrak{u}_{1}
$$

and let $B_{1}$ be the corresponding subgroup of $B$. Then it is clear from (7.13) that $B_{1}$ acts simply-transitively on $\mathcal{O}^{f}$. We may use this observation to obtain canonical symplectic coordinates on $\mathcal{O}^{f}$ as follows:

Let $\left\{H_{1}, \ldots, H_{\ell}\right\}$ be the basis for a dual to $\left\{\alpha_{1}, \ldots, \alpha_{\ell}\right\}$. Define a diffeomorphism $\psi: \mathbb{R}^{\ell} \times \mathbb{R}^{\ell} \rightarrow \mathcal{O}\left(=\mathcal{O}^{f}\right)$ by

$$
\psi(p, q)=\exp \left(\sum_{i=1}^{\ell} q_{i} H_{i}\right) \exp \left(\sum_{i=1}^{\ell}\left(p_{i} / c_{i}\right) X_{i}\right) \cdot f .
$$

If we express the remaining roots $\alpha_{\ell+1}, \ldots, \alpha_{m}$ in terms of $\alpha_{1}, \ldots, \alpha_{\ell}$ by

$$
\alpha_{i}=\sum_{j=1}^{\ell} d_{i j} \alpha_{j}, \quad i>\ell,
$$

and define

$$
q_{i}=\sum_{j=1}^{\ell} d_{i j} q_{j}, \quad i>\ell,
$$

then

$$
\psi(p, q)=\sum_{i=1}^{\ell} p_{i} \alpha_{i}+\sum_{j=1}^{m} c_{j} e^{-q_{j}} X_{j}^{*}
$$


Viewing $p_{i}$ and $q_{j}$ as functions on $\mathcal{O}$, it is immediate from (7.15) that

$$
H_{H_{i}}^{\mathscr{O}}=p_{i}, H_{X_{j}}^{\mathscr{Q}}=c_{j} e^{-q_{j}} .
$$

Since $\left[H_{i}, X_{j}\right]=\delta_{i j} X_{j}$, the corresponding Poisson bracket relation is thus

$$
\left\{p_{i}, e^{-q_{j}}\right\}=\delta_{i j} e^{-q_{j}},
$$

by (7.16) and Lemma 7.3. Hence

$$
\left\{p_{i}, q_{j}\right\}=-\delta_{i j}
$$

[Recall that if $f$ and $g$ are real functions on a symplectic manifold, and $\phi$ is a smooth function defined on an open set containing the range of $g$, then $\{f, \phi(g)\}=\phi^{\prime}(g)\{f, g\}$.] Similarly, one has $\left\{p_{i}, p_{j}\right\}=0$ and $\left\{q_{i}, q_{j}\right\}=0$. Thus $q_{1}, \ldots, q_{\ell}$, $p_{1}, \ldots, p_{\ell}$ is a set of canonical symplectic coordinates on $\mathcal{O}$.

With $\left\{H_{i}\right\}$ as above, the Laplacian of $\mathfrak{b}$ is

$$
\Omega=\sum_{i, j=1}^{\ell}\left\langle\alpha_{i}, \alpha_{j}\right\rangle H_{i} H_{j}+\sum_{j=1}^{m} X_{j}^{2} .
$$

It follows from (7.16) that the corresponding Hamiltonian function $j(\Omega)$ on $\mathcal{O}$ is

$$
\sum_{i, j=1}^{\ell}\left\langle\alpha_{i}, \alpha_{j}\right\rangle p_{i} p_{j}+\sum_{j=1}^{m} c_{j}^{2} e^{-2 q_{j}} .
$$

Note that the choice of $\mathcal{O}$ determines the magnitude of the coefficients $c_{i}^{2}$ in the "potential energy" term, while the geometry of the set of roots (relative to the given inner product on a) determines the "kinetic energy" term.

By means of a canonical transformation we can put all the geometry of the system into the potential energy term. Let $\left\{\varepsilon_{i}\right\}$ be an orthonormal basis for $\mathfrak{a}^{*}$, and assume that

$$
\alpha_{i}=\sum_{j=1}^{\ell} b_{i j} \varepsilon_{j}, \quad 1 \leqq i \leqq m .
$$

Make the canonical coordinate transformation

$$
x_{j}=\sum_{i=1}^{\ell} b_{i j} p_{i}, y_{i}=\sum_{j=1}^{\ell} b^{i j} q_{j}
$$

where $\left[b^{i j}\right]_{1 \leqq i, j \leqq \ell}$ is the inverse matrix to $\left[b_{i j}\right]_{1 \leqq i, j \leqq \ell}$. Then $x_{1}, \ldots, x_{\ell}, y_{1}, \ldots, y_{\ell}$ are global symplectic coordinates on $\mathcal{O}$, and

$$
j(\Omega)^{\mathcal{O}}=\sum_{i=1}^{\ell} x_{i}^{2}+\sum_{i=1}^{m} c_{i}^{2} \exp \left(-2 \sum_{j=1}^{\ell} b_{i j} y_{j}\right) .
$$

In these coordinates one sees that $j(\Omega)$ is a Hamiltonian of "generalized Toda Lattice" type. (For more explicit formulas, cf. [12] for the case of Dynkin diagrams, and $[2,5]$ for completed Dynkin diagrams.)

\section{Lax Forms}

Let $G$ be a linear, connected semi-simple Lie group, with Lie algebra $\mathfrak{g}$ and Iwasawa decomposition $G=K A N,(\mathfrak{g}=\mathfrak{t}+\mathfrak{a}+\mathfrak{n})$ as in Sect. 1 . We assume that $G$ is split over $\mathbb{R}$, and has rank $\ell>1$. Let $\theta: \mathfrak{g} \rightarrow \mathfrak{g}$ be the Cartan involution associated 
with $\mathfrak{f}$, and put $\overline{\mathfrak{n}}=\theta(\mathfrak{n})$. Since $\mathfrak{g}$ is split, one has $\mathfrak{g}=\overline{\mathfrak{n}}+\mathfrak{a}+\mathfrak{n}$. If $X \in \mathfrak{g}$, write $X=X_{+}+X_{\mathfrak{a}}+X_{-}$, where $X_{+} \in \mathfrak{n}, X_{-} \in \overline{\mathfrak{n}}$, and $X_{\mathfrak{a}} \in \mathfrak{a}$. Let $\mathfrak{p}=\{X \in \mathfrak{g}: \theta X=-X\}$. Then $X \in \mathfrak{p}$ if and only if $\theta\left(X_{+}\right)=-X_{-}$.

Let $\Delta^{+}$be the set of roots of $a$ on $n$. Let $\pi=\left\{\alpha_{1}, \ldots, \alpha_{\ell}\right\}$ be the simple roots in $\Delta^{+}$, and let $\Delta^{+}=\left\{\alpha_{1}, \ldots, \alpha_{d}\right\}$, with $\alpha_{1}<\alpha_{2}<\ldots<\alpha_{d}$ relative to a lexicographic order on $\mathfrak{a}^{*}$.

Let $Z_{i} \in \mathfrak{n}_{\alpha_{i}}$ be such that $-B\left(Z_{i}, \theta Z_{i}\right)=\delta_{i j}$. Put $X_{i}=Z_{i}-\theta Z_{i}, Y_{i}=Z_{i}+\theta Z_{i}$. Then

$$
\begin{aligned}
\mathfrak{l} & =\sum_{i=1}^{d} \mathbb{R} Y_{i}, \mathfrak{p}=\mathfrak{a} \oplus \sum_{i=1}^{d} \mathbb{R} X_{i}, \\
B\left(Y_{i}, Y_{j}\right) & =-2 \delta_{i j}, B\left(X_{i}, X_{j}\right)=2 \delta_{i j}
\end{aligned}
$$

( $B$ the Cartan-Killing form).

The set $\left\{\alpha_{1}, \ldots, \alpha_{\ell},-\alpha_{d}\right\}$ corresponds to a completed Dynkin diagram in Bourbaki's terminology [6], and is an extended Dynkin diagram, in the sense of Definition 5.1. We are assuming $\ell>1$, so that $\alpha_{d}$ is not a simple root. Let $\mathfrak{b}=\mathfrak{a} \oplus \mathfrak{u}$ be the corresponding generalized " $a x+b$ " algebra, as in Sect. 2. Here we label a basis for $\mathfrak{u}$ as $\bar{Z}_{1}, \ldots, \bar{Z}_{\ell}, \bar{Z}_{d}$, with the only non-trivial commutation relations in $\mathfrak{b}$ being

$$
\begin{aligned}
{\left[H, \bar{Z}_{i}\right] } & =\alpha_{i}(H) \bar{Z}_{i}, \quad 1 \leqq i \leqq \ell \\
{\left[H, \bar{Z}_{d}\right] } & =-\alpha_{d}(H) \bar{Z}_{d}
\end{aligned}
$$

for $H \in \mathfrak{a}$. On $\mathfrak{b}$ we put the inner product, $\langle\cdot, \cdot\rangle$, defined by

$$
\begin{aligned}
& \left.\langle\cdot, \cdot\rangle\right|_{\mathfrak{a} \times \mathbf{a}}=\left.B\right|_{a \times a} \\
& \left\langle\bar{Z}_{i}, \bar{Z}_{j}\right\rangle=(1 / 2) \delta_{i j} .
\end{aligned}
$$

Give $\mathfrak{b}^{*}$ the inner product dual to the inner product on $\mathfrak{b}$. For $f \in \mathfrak{b}^{*}$, define $f^{b} \in \mathfrak{b}$ by

$$
\left\langle f^{b}, X\right\rangle=f(X), X \in \mathfrak{b} .
$$

Thus for $f_{1}, f_{2} \in \mathfrak{b}^{*}$, one has $f_{1}^{\mathfrak{b}} \cdot f_{2} \in \mathfrak{b}^{*}$ given by $f_{1}^{b} \cdot f_{2}(X)=f_{2}\left(\left[X, f_{1}^{b}\right]\right), X \in \mathfrak{b}$. For $X \in \mathfrak{b}$ define $X^{\#} \in \mathfrak{b}^{*}$ by $X^{\#}(Y)=\langle X, Y\rangle, Y \in \mathfrak{b}$.

In this section we show that every $K$-invariant smooth function on $\mathfrak{p}$ defines a function on $\mathfrak{b}^{*}$ which Poisson-commutes with the Laplacian of $\mathfrak{b}$. To do this, we generalize Flaschka's construction of Lax forms [7], as follows (van Moerbeke [19]):

Define the subspace

$$
\mathfrak{p}_{1}=\mathfrak{a} \oplus \sum_{i=1}^{\ell} \mathbb{R} X_{i} \oplus \mathbb{R} X_{d}
$$

of $\mathfrak{p}$. For example, when $\mathfrak{g}=\mathrm{s} \ell(n, \mathbb{R}), n \geqq 3$, and $\mathfrak{p}$ is taken as the trace zero symmetric matrices, then $\mathfrak{p}_{1}$ consists of all "periodic Jacobi matrices" of trace zero:

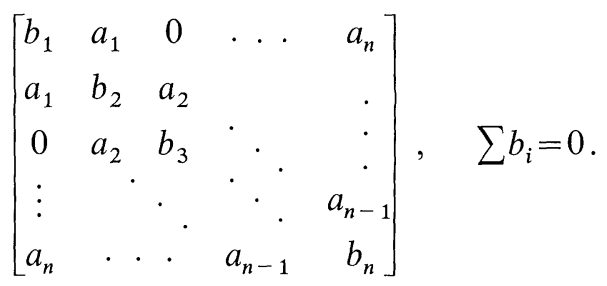


Let $\bar{Z}_{i}^{*}$, for $1 \leqq i \leqq \ell$ and $i=d$, be the linear functionals on $\mathfrak{b}$ defined by

$$
\bar{Z}_{i}^{*}\left(H+\sum c_{k} \bar{Z}_{k}\right)=c_{i}, \quad \text { for } \quad H \in \mathfrak{a} .
$$

Introduce a linear map $F: \mathfrak{p} \rightarrow \mathfrak{b}^{*}$ by

$$
F\left(H+\sum_{i=1}^{d} c_{i} X_{i}\right)=H^{\#}+\sum_{i=1}^{\ell} c_{i} \bar{Z}_{i}^{*}+c_{d} \bar{Z}_{d}^{*} .
$$

Since $\left\langle\bar{Z}_{i}^{*}, \bar{Z}_{j}^{*}\right\rangle=2 \delta_{i j}$, it is clear that $\left.F\right|_{\mathfrak{p}_{1}}$ is an isometry.

Denote by $C_{\mathbb{R}}^{\infty}(\mathfrak{p})^{K}$ the $\operatorname{Ad}(K)$-invariant smooth real-valued functions on $\mathfrak{p}$. For a real $C^{1}$ function $\phi$ on $\mathfrak{p}$, let $\nabla \phi$ denote the gradient of $\phi$, relative to the inner product given by the Cartan-Killing form. Let $s_{d}: \mathfrak{p} \rightarrow \mathfrak{p}$ be the reflection about the hyperplane $\left(X_{d}\right)^{\perp}$. That is,

$$
s_{d}(X)=X-B\left(X, X_{d}\right) X_{d} .
$$

Lemma 8.1. If $\phi \in C_{\mathbb{R}}^{\infty}(\mathfrak{p})^{K}$, then $[\nabla \phi(X), X]=0$ for $X \in \mathfrak{p}$.

Proof. Since $\operatorname{Ad}(k)$ acts orthogonally on $\mathfrak{p}$, for any $k \in K$, one has

$$
\phi(\operatorname{Ad}(k) X)=\operatorname{Ad}(k)(\nabla \phi(X)) .
$$

But $\operatorname{Ad}(k)$ is also a Lie algebra automorphism, so

$$
[\nabla \phi(\operatorname{Ad}(k) X), \operatorname{Ad}(k) X]=\operatorname{Ad}(k)[\nabla \phi(X), X] .
$$

Thus to prove the lemma, it suffices to take $X \in \mathfrak{a}$, since $\mathfrak{p}=\operatorname{Ad}(K) \mathfrak{a}$.

We may assume, by continuity, that $X$ is regular, so that

$$
\mathfrak{a}=\{Z \in \mathfrak{p}:[Z, X]=0\} .
$$

In this case, one has

$$
\mathfrak{a}=(\operatorname{ad}(\mathfrak{l}) X)^{\perp} .
$$

Indeed, if $Z \in \mathfrak{p}$ and $Z \perp[Y, X]$ for all $Y \in \mathfrak{f}$, then $0=B(Z,[Y, X])=B([X, Z], Y)$. Since $[X, Z] \in \mathfrak{f}$, it follows that $[X, Z]=0$, and hence $Z \in \mathfrak{a}$. Reversing the argument gives the opposite inclusion and proves $(*)$.

Now take $Z=\nabla \phi(X)$. Since $\phi$ is constant on $K$ orbits, $Z$ is orthogonal to the $K$ orbit through $X$, and hence $Z \in \mathfrak{a}$, by (8.3), Q.E.D.

Lemma 8.2. Let $X \in \mathfrak{p}_{1}$ and $Y \in \mathfrak{p}$. Then

$$
F(Y)^{b} \cdot F(X)=F\left(\left[Y_{+}-Y_{-}, s_{d} X\right]_{\mathfrak{a}}-s_{d}\left[Y_{a}, X_{+}-X_{-}\right]\right) .
$$

Proof. Assume that $X=X_{a}+\sum b_{i} X_{i}$ and $Y=Y_{a}+\sum c_{i} X_{i}$, where $b_{i}=0$ for $\ell<i<d$. Then by (8.2),

$$
F(Y)^{b}=Y_{\mathfrak{a}}+2 \sum_{i=1}^{\ell} c_{i} \bar{Z}_{i}+2 c_{d} \bar{Z}_{d} .
$$

Formulas (7.12) for the coadjoint action of $\mathfrak{b}$ imply that

$$
\begin{aligned}
F(Y)^{b} \cdot F(X)= & -\sum_{i=1}^{\ell} b_{i} \alpha_{i}\left(Y_{\alpha}\right) \bar{Z}_{i}^{*} \\
& +b_{d} \alpha_{d}\left(Y_{a}\right) \bar{Z}_{d}^{*}+2 \sum_{i=1}^{\ell} b_{i} c_{i} \alpha_{i}-2 b_{d} c_{d} \alpha_{d} .
\end{aligned}
$$


On the other hand, $X_{+}-X_{-}=\sum b_{i} Y_{i}$, and for $H \in \mathfrak{a}$ one has

$$
\begin{aligned}
{\left[H, X_{i}\right] } & =\alpha_{i}(H) Y_{i} \\
{\left[H, Y_{i}\right] } & =\alpha_{i}(H) X_{i} .
\end{aligned}
$$

Thus $\left[Y_{\mathfrak{a}}, X_{+}-X_{-}\right]=\sum b_{i} \alpha_{i}\left(Y_{\mathfrak{a}}\right) X_{i}$, so that

$$
F\left(s_{d}\left[Y_{\mathfrak{a}}, X_{+}-X_{-}\right]\right)=\sum_{i=1}^{\ell} b_{i} \alpha_{i}\left(Y_{\mathfrak{a}}\right) \bar{Z}_{i}^{*}-b_{d} \alpha_{d}\left(Y_{\mathfrak{a}}\right) \bar{Z}_{d}^{*} .
$$

Furthermore, for $H \in \mathfrak{a}$, one has

$$
B\left(H,\left[Y_{i}, X_{j}\right]\right)=-B\left(\left[H, X_{j}\right], Y_{i}\right)=-2 \alpha_{j}(H) B\left(Y_{j}, Y_{i}\right)=2 \delta_{i j} \alpha_{j}(H)
$$

by (8.5), so that $\left[Y_{j}, X_{i}\right]_{\mathfrak{a}}=2 \delta_{i j} H_{\alpha_{i}}$. It follows that $\left[Y_{+}-Y_{-}, X\right]_{\mathfrak{a}}$ $=\sum b_{i} c_{j}\left[Y_{j}, X_{i}\right]_{\mathfrak{a}}=2 \sum b_{i} c_{i} H_{\alpha_{i}}$, and thus

$$
F\left(\left[Y_{+}-Y_{-}, s_{d} X\right]_{\mathfrak{a}}\right)=2 \sum_{i=1}^{\ell} b_{i} c_{i} \alpha_{i}-2 b_{d} c_{d} \alpha_{d} .
$$

Using (8.6) and (8.7) in (8.4), we obtain (8.3), Q.E.D.

To obtain the Lax forms from the two preceding lemmas, we need the following calculation, which uses the maximality of the root $\alpha_{d}$ for the first time:

Lemma 8.3. Suppose $X \in \mathfrak{p}_{1}, Y \in \mathfrak{p}$, and $[X, Y]=0$. Then

$$
\left[Y_{+}-Y_{-}, s_{d} X\right]_{-}=-\left[Y_{a}, X_{+}-X_{-}\right]_{-} .
$$

Proof. It suffices to calculate all commutators $\bmod \mathfrak{s}$. Write $X=X^{\prime}+c X_{d}$, where $X^{\prime} \in \operatorname{span}\left\{X_{i} ; 1 \leqq i \leqq \ell\right\}$. Then $\left[Y_{+}, X^{\prime}\right] \in \mathfrak{s}$, so that

$$
\left[Y_{+}, X\right] \equiv\left[Y_{+}, c X_{d}\right] \equiv-\left[Y_{+}, s_{d} X\right]
$$

$\bmod (\mathfrak{s})$. Furthermore $\left[Y_{-}, \theta Z_{d}\right]=0$ and $\left[Y_{-}, Z_{d}\right] \in \mathfrak{s}$, since $\alpha_{d}$ is maximal. Thus

$$
\left[Y_{-}, X\right] \equiv\left[Y_{-}, X^{\prime}\right] \equiv\left[Y_{-}, s_{d} X\right]
$$

$\bmod (\mathfrak{s})$.

Now to verify $(8.8)$, note that $-\left[Y_{\mathfrak{a}}, X_{+}-X_{-}\right] \equiv\left[Y_{\mathfrak{a}}, X_{+}+X_{-}\right] \equiv\left[Y_{\mathfrak{a}}, X\right]$ $\bmod (\mathfrak{s})$. But $[Y, X]=0$ implies that

$$
\left[Y_{a}, X\right]=-\left[Y_{+}+Y_{-}, X\right] .
$$

Hence by (8.9) and (8.10),

$$
-\left[Y_{\mathfrak{a}}, X_{+}-X_{-}\right] \equiv-\left[Y_{+}+Y_{-}, X\right] \equiv\left[Y_{+}-Y_{-}, s_{d} X\right]
$$

$\bmod (\mathfrak{s})$, Q.E.D.

Lemma 8.4. Suppose $X \in \mathfrak{p}_{1}, Y \in \mathfrak{p}$, and $[X, Y]=0$. Then $\left[Y_{+}-Y_{-}, X\right] \in \mathfrak{p}_{1}$, and

$$
F\left(s_{d}\left[Y_{+}-Y_{-}, s_{d} X\right]\right)=F(Y)^{b} \cdot F(X) .
$$

Proof. This follows immediately from Lemmas 8.2 and 8.3 , since $\left[Y_{\mathfrak{a}}, X_{+}-X_{-}\right]_{\mathfrak{a}}=0$ and $\left[Y_{\mathfrak{a}}, X_{+}-X_{-}\right]_{+}=\left[Y_{+}-Y_{-}, s_{d} X\right]_{+}$by (8.8).

We come now to the main result of this section. If $\phi$ is a function on $\mathfrak{p}$, define a function $w_{\phi}$ on $\mathfrak{b}^{*}$ by

$$
w_{\phi}(F(X))=\phi\left(s_{d} X\right), \quad X \in \mathfrak{p}_{1} .
$$


Define a vector field $L \phi$ on $\mathfrak{p}$ by

$$
L_{X}^{\phi}=\left[\nabla \phi\left(s_{d} X\right)_{+}-\nabla \phi\left(s_{d} X\right)_{-}, X\right],
$$

for $X \in p$.

Theorem 8.5. Suppose $\phi \in C_{\mathbb{R}}^{\infty}(\mathfrak{p})^{K}$. Then for $X \in \mathfrak{p}_{1}$,

$$
d w_{\phi}(F(X)) \cdot F(X)=F\left(L_{X}^{\phi}\right) .
$$

Remarks. Recalling the results of Sect. 7 concerning left-invariant Hamiltonian vector fields on $T^{*}(B)$ ( $B$ the simply-connected Lie group with Lie algebra $b$ ), we see that $L^{\phi}$ is a "Lax form" for the field with Hamiltonian $w_{\phi}$. For the case $\phi(X)=\langle X, X\rangle$ and $G=\mathrm{SL}(n, \mathbb{R})$, Eq. (8.13) was first observed by Flaschka [7].

Proof. Since the map $F$ is linear and isometric on $\mathfrak{p}_{1}$, one has

$$
d w_{\phi}(F(X))=F\left(s_{d} \nabla \phi\left(s_{d} X\right)\right)^{b} .
$$

Let $\sigma$ be the automorphism of $\mathfrak{b}$ which fixes $\mathfrak{a}$ and $\bar{Z}_{i}, 1 \leqq i \leqq \ell$, and sends $\bar{Z}_{d}$ to $-\bar{Z}_{d}$. Then $F\left(s_{d} Y\right)=\sigma^{*}(F(Y))$, and $\sigma^{*}(Z \cdot f)=(\sigma Z) \cdot \sigma^{*} f$ for $Z \in \mathfrak{b}, f \in b^{*}$. Thus

$$
d w_{\phi}(F(X)) \cdot F(X)=\sigma^{*}\left(F\left(\nabla \phi\left(s_{d} X\right)\right)^{b} \cdot F\left(s_{d} X\right)\right) .
$$

By Lemmas 8.1 and 8.4 , this gives

$$
d w_{\phi}(F(x)) \cdot F(X)=\sigma^{*} F\left(s_{d}\left[\nabla \phi\left(s_{d} X\right)_{+}-\nabla \phi\left(s_{d} X\right)_{-}, X\right]\right)=F\left(L_{X}^{\phi}\right) .
$$

Corollary 8.6. Suppose $\phi, \psi \in C_{\mathbb{R}}^{\infty}(\mathfrak{p})^{K}$. Set $\psi^{\iota}=\psi \circ S_{d}$. Then $w_{\phi}$ and $w_{\psi^{-}}$Poissoncommute on $\mathfrak{b}^{*}$. In particular, $w_{\phi}$ Poisson-commutes with the Laplacian of $\mathfrak{b}$.

Proof. Since $w_{\psi^{\circ}} \circ F=\psi$ and $\left.F\right|_{\mathfrak{p}_{1}}$ is a linear isomorphism onto $\mathfrak{b}^{*}$, it suffices by Lemma 7.3 and Theorem 8.5 to show that $L_{X}^{\phi}(\psi)=0$ for $X \in p_{1}$. Now if we set $A(X)=\operatorname{ad}\left(\nabla \phi\left(s_{d} X\right)_{+}-\nabla \phi\left(s_{d} X\right)_{-}\right)$, then

$$
\begin{aligned}
L_{X}^{\phi}(\psi) & =\left.\frac{d}{d t} \psi(X+t A(X)(X))\right|_{t=0} \\
& =\left.\frac{d}{d t} \psi\left(e^{t A(X)}(X)\right)\right|_{t=0}=0,
\end{aligned}
$$

since $A(X) \in \operatorname{ad}(\mathfrak{l})$.

The Laplacian on $\mathfrak{b}$ corresponds to the Hamiltonian $w_{\psi}$, where $\psi(X)=\langle X, X\rangle$ on $\mathfrak{p}$. Since $\psi \circ S_{d}=\psi$ in this case, it follows that $\left\{w_{\psi}, w_{\phi}\right\}=0$, Q.E.D.

\section{Complete Integrability via Lax Forms}

We continue the hypotheses and notation of Sect. 8. Let $\Omega$ be the Laplacian of $\mathfrak{b}$, $j: U(\mathfrak{b}) \rightarrow S(\mathfrak{b})$ the symmetrization map. Then viewed as a function on $\mathfrak{b}^{*}, j(\Omega)=w_{\psi}$, where $\psi(X)=\langle X, X\rangle, X \in \mathfrak{p}$.

Theorem 9.1. Let $\phi_{1}, \ldots, \phi_{\ell}$ be a set of algebraically independent generators for $S\left(\mathfrak{p}^{*}\right)^{K}$. Let

$$
\Xi=\bar{Z}_{1}^{n_{1}} \ldots \bar{Z}_{\ell}^{n_{\ell}} \bar{Z}_{d}
$$


where $\left\{\bar{Z}_{i}\right\}$ satisfy (8.1) and $\alpha_{d}=\sum_{i=1}^{\ell} n_{i} \alpha_{i}$. Then $S(\mathfrak{b})^{j(\Omega)}$ is a polynomial ring, and is generated by the elements $w_{\phi_{1}}, \ldots, w_{\phi_{\ell}}$ and $\Xi$, which mutually Poisson-commute on $\mathfrak{b}^{*}$. In particular, $\left\{w_{\phi}, w_{\psi}\right\}=0$ for all $K$-invariant polynomials $\phi$ and $\psi$ on $\mathfrak{p}$.

Remark. To compare this result with Theorem 6.4, recall that we are here allowing any completed Dynkin diagram for the set of roots of $\mathfrak{b}$. In particular, this includes the completed diagrams of type $G_{2}, F_{4}, E_{7}$, and $E_{8}$, which were excluded in Theorem 6.4. For these exceptional diagrams, however, we do not know if the corresponding result holds in the enveloping algebra (Theorem 5.2). Also, we cannot treat the extended diagrams defined by a short dominant root using Lax forms.

Before proving Theorem 9.1, we need the Poisson algebra version of some results from Sect. 3. Let $\sigma \in \operatorname{Aut}(\mathfrak{b})$ be the reflection sending $\bar{Z}_{d}$ to $-\bar{Z}_{d}$ and fixing $\left(\bar{Z}_{d}\right)^{\perp}$. From the definition of the "Flaschka map" $F: \mathfrak{p} \rightarrow \mathfrak{b}^{*}$ it is clear that

$$
\sigma w_{\phi}=w_{\phi^{\prime}}, \text { for } \phi \in S\left(\mathfrak{p}^{*}\right) \text {. }
$$

Here we have extended $\sigma$ to an automorphism of $S(\mathfrak{b})$. Note that since $\sigma$ is a Liealgebra automorphism of $\mathfrak{b}$, its extension to $S(\mathfrak{b})$ is a Poisson-algebra automorphism. Since $\sigma \Omega=\Omega$, it follows that

$$
\sigma: S(\mathfrak{b})^{j(\Omega)} \rightarrow S(\mathfrak{b})^{j(\Omega)}
$$

Lemma 9.2. If $\phi \in S\left(\mathfrak{p}^{*}\right)^{K}$, then $\sigma w_{\phi}-w_{\phi}$ is divisible by $\xi$.

Proof. Let $v: S(\mathfrak{b})^{j(\Omega)} \rightarrow S(\mathfrak{a})$ be the map as in diagram (6.3). Since $v \circ \sigma=v$, one has $\sigma w_{\phi}-w_{\phi} \in \operatorname{Ker}(v)$. Now use the same argument as in Lemma 3.5, but with Lie brackets replaced by Poisson brackets. The details are left to the reader.

Lemma 9.3. Let $h$ be the Coxeter number of the Dynkin diagram $\pi$, and let $\phi, \psi \in S\left(p^{*}\right)^{K}$.

(i) If $\operatorname{deg} \phi<h$, then $\sigma w_{\phi}=w_{\phi}$.

(ii) If $\operatorname{deg} \phi=h$, then $\sigma w_{\phi}=w_{\phi}+c \Xi$ for some $c \in \mathbb{R}$.

(iii) If $\operatorname{deg} \phi \leqq h$ and $\operatorname{deg} \psi \leqq h$, then $\left\{w_{\phi}, w_{\psi}\right\}=0$.

Proof. As before, we use the fact that $h=1+\sum_{i=1}^{\ell} n_{i}=\operatorname{deg} \Xi$, and $\Xi$ Poisson commutes with any function on $\mathfrak{b}^{*}$. Thus (i) and (ii) follow immediately from Lemma 9.2. As for (iii), note that $\left\{w_{\phi}, w_{\psi}\right\}=\left\{w_{\phi}, w_{\psi}\right\}$ by (i), (ii) and (9.2). But this bracket vanishes, by Corollary 8.6.

Proof of Theorem 9.1. The existence of generators $\phi_{1}, \ldots, \phi_{\ell}$ for $S\left(p^{*}\right)^{K}$ is given by Chevalley's theorem [9], which also asserts that the restriction mapping to $\mathfrak{a}$ is an isomorphism onto $S\left(\mathfrak{a}^{*}\right)^{W}$. Thus $w_{\phi_{1}}, \ldots, w_{\phi_{\ell}}, \Xi$ are algebraically independent.

One knows that the Coxeter number $h=\max \left(\operatorname{deg} \phi_{j}\right)$, so by Lemma 9.3 $w_{\phi_{1}}, \ldots, w_{\phi_{\ell}}, \Xi$ mutually Poisson-commute. The proof that they generate $S(\mathfrak{b})^{j(\Omega)}$ is then identical to the proof of Theorem 6.4, part (a), since by Chevalley's theorem $v: \mathbb{R}\left[w_{\phi_{1}}, \ldots, w_{\phi_{\ell}}\right] \rightarrow S(\mathfrak{a})^{W}$ is surjective. This proves the theorem.

Corollary 9.4. Suppose $\phi \in S\left(\mathfrak{p}^{*}\right)^{K}$ and $\operatorname{deg} \phi \leqq h$, the Coxeter number of the Dynkin diagram $\pi$. Then for $f \in \mathfrak{b}^{*}$,

$$
d w_{\phi}(f) \cdot f=d w_{\phi^{\ulcorner}}(f) \cdot f .
$$


Proof. By Lemma 9.3, $d w_{\phi^{\circ}}=d w_{\phi}+c d \Xi$. But $\Xi$ is constant on $B$-orbits in $\mathfrak{b}^{*}$, so $d \Xi(f) \cdot f=0$, which yields the result.

Remarks on "Complete Integrability". Let $\mathcal{O} \subset \mathfrak{b} *$ be a generic orbit. From Sect. 7 we know that $\operatorname{dim} \mathcal{O}=2 \ell$. The function $\Xi$ is constant on $\mathcal{O}$, since $\Xi=j(\xi)$ with $\xi$ in the center of $U(\mathfrak{b})$. The remaining generators $w_{\phi_{1}}, \ldots, w_{\phi_{\ell}}$ of $S(\mathfrak{b})^{j(\Omega)}$ are easily seen to be functionally independent at "generic" points of $\mathcal{O}$. Indeed, if $\psi: \mathbb{R}^{\ell} \times \mathbb{R}^{\ell} \rightarrow \mathcal{O}$ is the map in (7.15), and we set

$$
H(p)=\sum_{i=1}^{\ell} p_{i} H_{i}, X(q)=\sum_{i=1}^{\ell} c_{i} e^{-q_{i}} X_{i},
$$

then $\psi(p, q)=F(H(p)+X(q))$ (notation as in Sect. 8). Hence for $\phi \in C_{\mathbb{R}}^{\infty}(p)$, one has

$$
w_{\phi}(\psi(p, q))=\phi(H(p)+X(q)) .
$$

Now $X(q) \rightarrow 0$ if $q_{i} \rightarrow+\infty$ for all $i$, and the basic invariants $\phi_{1}, \ldots, \phi_{\ell}$ are known to be functionally independent at regular points of $\mathfrak{a}[6, \mathrm{p} .113]$. It follows then from (9.3) that $\partial\left(w_{\phi_{1}}, \ldots, w_{\phi_{\ell}}\right) / \partial\left(p_{1}, \ldots, p_{\ell}\right)$ has rank $\ell$ on a non-empty open subset of $\mathcal{O}$, giving generic functional independence.

\section{References}

1. Adler, M.: Invent. Math. 50, 219-248 (1979)

2. Adler, M., van Moerbeke, P.: Adv. Math. 38, 267-317 (1980)

3. Arnol'd, V.: Ann. Inst. Fourier (Grenoble) 16, 319-361 (1966)

4. Arnol'd, V.: Mathematical methods of classical mechanics. Berlin, Heidelberg, New York: Springer 1978

5. Bogoyavlensky, O.I. : Commun. Math. Phys. 51, 201-209 (1976)

6. Bourbaki, N.: Groupes et algèbres de Lie, Chaps. IV-VI (Éléments de mathématique, Fasc. XXXIV). Paris: Hermann 1968

7. Flaschka, H.: Phys. Rev. B9, 1924-1925 (1974)

8. Goodman, R., Wallach, N.R.: J. Funct. Anal. 39, 199-279 (1980)

9. Helgason, S.: Differential geometry and symmetric spaces. New York: Academic Press 1962

10. Hénon, M.: Phys. Rev. B9, 1921-1923 (1974)

11. Kostant, B.: Invent. Math. 48, 101-184 (1978)

12. Kostant, B.: Adv. Math. 34, 195-338 (1979)

13. Moser, J.: Various aspects of integrable Hamiltonian systems. In: Dynamical systems. Progress in Mathematics, Vol. 8. Boston: Birkhäuser 1980

14. Miščenko, A.S., Fomenko, A.T.: Izv. Akad. Nauk. SSSR Ser. Mat. 42, 396-415 (1978); Math. USSR Izv. 12, 371-389 (1978)

15. Nelson, E., Stinespring, W.F.: Am. J. Math. 81, 547-560 (1959)

16. Olshanetsky, M.A., Perelomov, A.M.: Invent. Math. 54, 261-269 (1979)

17. Ratiu, T.: Involution theorems. In: Geometric methods in mathematical physics. Lecture Notes in Mathematics, Vol. 775. Berlin, Heidelberg, New York: Springer 1980

18. Vergne, M.: Bull. Soc. Math. Fr. 100, 301-335 (1972)

19. van Moerbeke, P.: Invent. Math. 37, 45-81 (1976)

20. Wallach, N.R.: Symplectic geometry and fourier analysis. Lie Groups: History, Frontiers, and Applications. V. Brookline, Massachusetts: Math. Sci. Press 1977

Communicated by A. Jaffe

Received May 12, 1981

Note added in proof. B. Kostant has informed us that he has also obtained a finite dimensional Lax form for generalized periodic Toda lattices ["Poisson Commutativity and the Generalized Periodic Toda Lattice" (preprint)]. 